\title{
STATIONARY HARMONIC MEASURE AND DLA IN THE UPPER HALF PLANE
}

\author{
EVIATAR B. PROCACCIA AND YUAN ZHANG
}

\begin{abstract}
In this paper, we introduce the stationary harmonic measure in the upper half plane. By bounding this measure, we are able to define both the discrete and continuous time diffusion limit aggregation (DLA) in the upper half plane with absorbing boundary conditions. We prove that for the continuous model the growth rate is bounded from above by $o\left(t^{2+\epsilon}\right)$. When time is discrete, we also prove a better upper bound of $o\left(n^{2 / 3+\epsilon}\right)$, on the maximum height of the aggregate at time $n$. An important tool developed in this paper, is an interface growth process, bounding any process growing according to the stationary harmonic measure. Together with [10] one obtains non zero growth rate for any such process.
\end{abstract}

\section{Contents}

1. Introduction 1

2. Properties of stationary harmonic measure 6

3. Uniform upper bounds on harmonic measure 9

4. Subset maximizing the stationary harmonic measure 16

5. Total harmonic measure on finite sets 26

6. Construction and growth estimate of DLA in $\mathcal{H}$

Acknowledgments 46

References $\quad 46$

\section{INTRODUCTION}

In this paper, we consider the stationary harmonic measure in the upper half plane and the corresponding diffusion limit aggregation (DLA). The Diffusion Limited Aggregation (DLA) in $\mathbb{Z}^{2}$ was introduced in 1983 by Witten and Sander [12] as a simple model to study the geometry and dynamics of physical systems governed by diffusive laws. The DLA is defined recursively as a process on subsets $A_{n} \in \mathbb{Z}^{2}$. Starting from $A_{0}=\{(0,0)\}$, at each time a new point $a_{n+1}$ sampled from the harmonic probability measure on $\partial^{\text {out }} A_{n}$ is added to $A_{n}$. Intuitively, $a_{n+1}$ is the first place that a random walk starting from infinity visits $\partial^{\text {out }} A_{n}$.

Although DLA is straightforwardly defined and easily simulated on a computer, very little about it is known rigorously. One of the notable exceptions is shown 
by Kesten where a polynomial upper bound, which equal to $n^{2 / 3}$ when $d=2$ and $n^{2 / d}$ when $d \geq 3$, of the growth rate on DLA arms is given, see Corollary in [7] or Theorem in [8]. In a later work [9] Kesten improved the upper bound for DLA when $d=3$ to $\sqrt{n \log (n)}$. No non-trivial lower bounds have been proved till present day. It is in fact still open to rule out that the DLA converges to a ball, although numerical simulations clearly exclude this.

Recently, this topic is re-visited by Benjamini and Yadin [2] where they "clean up' Kesten's argument, and make it more robust". They proved upper bounds on the growth rates of DLA's on "transitive graphs of polynomial growth, graphs of exponential growth, non-amenable graphs, super-critical percolation on $Z^{d}$ and high dimensional pre-Sierpinski carpets".

In this paper, we further extend the reach of Kesten's idea to non-transitive graphs. We define the (horizontally) translation invariant stationary harmonic measure on the upper half plane with absorbing boundary condition and show the growth of such stationary harmonic measure in a connected subset intersecting $x$-axis is sub-linear with respect to the height. With the bounds found for our stationary harmonic measure, we will be able to define a continuous time DLA on the upper half plane and give upper bound on its growth rates.

We first define several sets and stopping times for our problem. Let $\mathcal{H}=$ $\left\{(x, y) \in \mathbb{Z}^{2}, y \geq 0\right\}$ be the upper half plane (including $x$-axis), and $S_{n}, n \geq 0$ be a 2-dimensional simple random walk. For any $x \in \mathbb{Z}^{2}$, we will write

$$
x=\left(x_{1}, x_{2}\right)
$$

with $x_{i}$ denote the $i$ th coordinate of $x$, and $\|x\|=\left|x_{1}\right|+\left|x_{2}\right|$. Then let $L_{n}, D_{n} \subset \mathbb{Z}^{2}$ be defined as follows: for each nonnegative integer $n$, define

$$
\begin{gathered}
L_{n}=\{(x, n), x \in \mathbb{Z}\}, \\
V_{n}=\{(0, k), 0 \leq k \leq n\},
\end{gathered}
$$

and

$$
U_{n}=L_{0} \cup V_{n}
$$

I.e., $L_{n}$ is the horizontal line of height $n$ while $U_{n}$ is $x$-axis plus the vertical line segment between $(0,0)$ and $(0, n)$. And we let $y_{n}=(0, n)$ be the "end point" of $V_{n}$. Moreover, we use $\mathcal{P}_{n} \subset \mathcal{H}$ for an arbitrary finite path in the upper half plane connecting $y_{n}$ and the $x$-axis. One can immediately see that $V_{n}$ is one such path.

And for each subset $A \subset \mathbb{Z}^{2}$ we define stopping times

$$
\bar{\tau}_{A}=\min \left\{n \geq 0, S_{n} \in A\right\}
$$

and

$$
\tau_{A}=\min \left\{n \geq \frac{1}{2}, S_{n} \in A\right\} .
$$


For any subsets $A_{1} \subset A_{2}$ and $B$ and any $y \in \mathbb{Z}^{2}$, by definition one can easily check that

$$
\begin{aligned}
& P_{y}\left(\tau_{A_{1}}<\tau_{B}\right) \leq P_{y}\left(\tau_{A_{2}}<\tau_{B}\right), \\
& P_{y}\left(\bar{\tau}_{A_{1}}<\bar{\tau}_{B}\right) \leq P_{y}\left(\bar{\tau}_{A_{2}}<\bar{\tau}_{B}\right),
\end{aligned}
$$

and that

$$
\begin{aligned}
& P_{y}\left(\tau_{B}<\tau_{A_{2}}\right) \leq P_{y}\left(\tau_{B}<\tau_{A_{1}}\right), \\
& P_{y}\left(\bar{\tau}_{B}<\bar{\tau}_{A_{2}}\right) \leq P_{y}\left(\bar{\tau}_{B}<\bar{\tau}_{A_{1}}\right),
\end{aligned}
$$

where $P_{y}(\cdot)=P\left(\cdot \mid S_{0}=y\right)$. Now we define the stationary harmonic measure on $\mathcal{H}$ which will serve as the Poisson intensity in our continuous time DLA model. For any connected $B \subset \mathcal{H}$, any edge $\vec{e}=x \rightarrow y$ with $x \in B, y \in \mathcal{H} \backslash B$ and any $N$, we define

$$
H_{B, N}(\vec{e})=\sum_{z \in L_{N} \backslash B} P_{z}\left(S_{\bar{\tau}_{B \cup L_{0}}}=x, S_{\bar{\tau}_{B \cup L_{0}}-1}=y\right)
$$

By definition, a necessary condition for $H_{B, N}(\vec{e})>0$ (although at this point we have not yet ruled out the possibility it equals to infinity) is $y \in \partial^{\text {out }} B$ and $|x-y|=$ 1. And for all $x \in B$, we can also define

$$
H_{B, N}(x)=\sum_{\vec{e} \text { starting from } x} H_{B, N}(\vec{e})=\sum_{z \in L_{N} \backslash B} P_{z}\left(S_{\bar{\tau}_{B \cup L_{0}}}=x\right) .
$$

And for each point $y \in \partial^{\text {out }} B$, we can also define

$$
\hat{H}_{B, N}(y)=\sum_{\substack{\vec{e} \text { starting in } B \\ \text { ending at } y}} H_{B, N}(\vec{e})=\sum_{z \in L_{N} \backslash B} P_{z}\left(\tau_{B} \leq \tau_{L_{0}}, S_{\bar{\tau}_{B \cup L_{0}}-1}=y\right) .
$$

By coupling and strong Markov property, we have $N \rightarrow H_{A, N}(e)$ is bounded and monotone in $N$. Thus

Proposition 1. For any $B$ and $\vec{e}$ as above, there is a finite $H_{B}(\vec{e})$ such that

$$
\lim _{N \rightarrow \infty} H_{B, N}(\vec{e})=H_{B}(\vec{e}) \text {. }
$$

And we call $H_{B}(\vec{e})$ the stationary harmonic measure of $\vec{e}$ with respect to $B$. Thus we immediately have the limits $H_{B}(x)=\lim _{N \rightarrow \infty} H_{B, N}(x)$ and $\hat{H}_{B}(y)=$ $\lim _{N \rightarrow \infty} \hat{H}_{B, N}(y)$ also exists and we call them the stationary harmonic measure of $x$ and $y$ with respect to $B$. Although now we have the limit $H_{B}(x)$ exists, it can be zero everywhere for certain $B$. We do not need to worry about this when $B$ is finite. For each finite $B$, we let

$$
H_{B}=\sum_{x \in B} H_{B}(x)=\sum_{y \in \partial^{\text {out }} B} \hat{H}_{B}(y)
$$

be the harmonic measure of $B$. Then we have $H_{B}$ is non-decreasing as $B$ gets larger: 
Proposition 2. For any finite subsets $B_{1} \subset B_{2} \subset \mathcal{H}$,

$$
H_{B_{2}} \geq H_{B_{1}} \text {. }
$$

Remark 1. However, for infinite subset of $\mathcal{H}$, it is possible to have the harmonic measure equal to 0 everywhere. In fact, we prove that as long as $B$ has a linear spatial growth horizontally, $H_{B}(\cdot)$ is uniformly 0 . On the other hand, we have also proved that for any $B$ with certain sub-linear spatial growth, it can have non-zero stationary harmonic measure. These results are presented in a separate paper [10].

After presenting the basic properties of our stationary harmonic measure, we can state our first main result which gives the following upper bounds on $H_{B, N}(x)$ :

Theorem 1. There is some constant $C<\infty$ such that for each connected $B \subset \mathcal{H}$ with $L_{0} \subset B$ and each $x=\left(x_{1}, x_{2}\right) \in B \backslash L_{0}$, and any $N$ sufficiently larger than $x_{2}$

$$
H_{B, N}(x) \leq C x_{2}^{1 / 2} \text {. }
$$

Remark 2. In this paper, we use $C$ and $c$ as constants in $(0, \infty)$ independent to the change of variables like $N$ or $n$. But their exact values can be different from place to place.

At the same time, we can also have the following result showing that for a point of height $n$, say $y_{n}$ without loss of generality, the harmonic measure is maximized (up to multiplying a constant) by $U_{n}$. I.e.

Theorem 2. There is some constant $c>0$ such that for all $N>n$,

$$
H_{U_{n}, N}\left(y_{n}\right) \geq c n^{1 / 2} \text {. }
$$

With Proposition 2, Theorem 1 and 2 and the bounds estimates in their proofs, we can further show that

Theorem 3. There are constants $0<c, C<\infty$ such that for any finite and connected $B$ in $\mathcal{H}$,

$$
H_{B} \leq C\left(\min _{x \in B}\left\{x_{2}\right\}+|B|\right)
$$

while

$$
\begin{gathered}
H_{B} \geq c \max _{x \in B}\left\{x_{2}\right\} \log ^{-1}\left(\max _{x \in B}\left\{x_{2}\right\}\right), \\
H_{B} \geq 1
\end{gathered}
$$

when $\max _{x \in B}\left\{x_{2}\right\}=0$.

And again, we also have the total harmonic measure is maximized (up to multiplying a constant) by the vertical line segment $V_{n}$ over all connected finite subsets with the same cardinality and intersecting $L_{0}$. 
Theorem 4. There is a constant $c>0$ such that for any $n$,

$$
H_{V_{n}} \geq c n \text {. }
$$

With the stationary harmonic measure bounded in Theorem 1, we are now able to define our DLA in the upper half plane as a continuous time stochastic process $A_{t}, t \geq 0$ taking values on finite subsets of $\mathcal{H}$. First we have $A_{0}=\{0\}$. For each $t \geq 0$. $A_{t}$ grows at a Poisson rate of $H_{A_{t}}$ and add a new point on $\partial^{\text {out }} A_{t}$ according to the probability distribution

$$
\tilde{p}\left(A_{t}, y\right)=\frac{\hat{H}_{A_{t}}(y)}{H_{A_{t}}}, y \in \mathcal{H}
$$

Similarly, we can also define the discrete DLA model $\left\{A_{n}\right\}_{n=0}^{\infty}$ in $\mathcal{H}$ which is the embedded Markov chain of $A_{t}$. I.e., at each $n, A_{n+1}=A_{n} \cup\{y\}$ where $y$ is sampled according to $\tilde{p}\left(A_{n}, y\right)$.

First, by introducing a pure growth interacting particle system that dominates the continuous time process, we show that $A_{t}$ is well defined and estimate an upper bound on the growth rate of its arms. For any finite $A$ define

$$
\|A\|=\max \{\|x\|, x \in A\} .
$$

Theorem 5. $A_{t}$ is well defined on $t \in[0, \infty)$. And for any $\epsilon>0$, we have with probability one

$$
\limsup _{t \rightarrow \infty} t^{-2-\epsilon}\left\|A_{t}\right\|=0
$$

Furthermore, we show that for any time $t,\left\|A_{t}\right\|$ has a finite $m$ th order moment for all $m \geq 1$.

Theorem 6. For any integer $m \geq 1$ and any $t \geq 0$

$$
E\left[\left\|A_{t}\right\|^{m}\right]<\infty \text {. }
$$

Remark 3. In our construction we are able to define the dominating interacting particle system starting from any initial configuration in $\{0,1\}^{\mathcal{H}}$, whose growth rate is given by the upper bound of the stationary harmonic measure found in Theorem 1. This, together with [10], allows us to define a horizontally translation invariant infinite $D L A$ on $\mathcal{H}$ and estimate its (non-zero) growth rate. We call this the stationary DLA model, and it will be presented in [11]. We refer the reader to look at recent results on other stationary aggregation processes $[1,3]$.

For the discrete time process let $h_{n}=\max _{x \in A_{n}}\left\{x_{2}\right\}$. By Theorem 1 and (10), we see that the probability that a new point $y$ is added to the aggregation $A_{n}$ is no larger than $\log \left(h_{n}\right) / \sqrt{h_{n}}$. Then the Borel-Cantelli argument in Step (ii) of [8] easily gives us a stronger upper bound on $h_{n}$ :

Theorem 7. For any $\epsilon>0$, we have with probability one

$$
\limsup _{n \rightarrow \infty} n_{5}^{-\epsilon-2 / 3} h_{n}=0 .
$$


The structure of this paper is as follows: In Section 2 we prove the more basic properties of the stationary harmonic measure, i.e., Proposition 1 and 2. Theorem 2 is proved in Section 3 by showing that certain arguments in [7] is actually sharp. Then we "inverse" the argument for vertical line segment and prove Theorem 2 in Section 4. In Section 5 we use the bounds found the the previous two sections and show Theorem 3 and 4 inductively. In Section 6, we use an interacting particle system argument to define the dominating process and prove Theorem 5 and 6 . After that, Theorem 7 follows immediately.

\section{Properties of Stationary harmonic measure}

2.1. Proof of Proposition 1. To show Proposition 1, we first need to verify that the infinite summation defined in (4) converges. Note that for $x_{2}>0$ and any $N>x_{2}$ and any $z \in L_{N} \backslash B$,

$$
P_{z}\left(S_{\bar{\tau}_{B}}=x\right)=\sum_{k=1}^{\infty} P_{z}\left(\bar{\tau}_{B \cup L_{0}}=k, S_{k}=x\right) .
$$

And by time reversal and symmetry of simple random walk, we have

$$
\begin{aligned}
P_{z}\left(\bar{\tau}_{B}=k, S_{k}=x\right) & =P_{z}\left(S_{k}=x, S_{1}, S_{2}, \cdots, S_{k-1} \notin B \cup L_{0}\right) \\
& =P_{x}\left(S_{k}=z, S_{1}, S_{2}, \cdots, S_{k-1} \notin B \cup L_{0}\right) \\
& =P_{x}\left(S_{k}=z, \tau_{B \cup L_{0}}>k\right) .
\end{aligned}
$$

Thus

$$
\begin{aligned}
P_{z}\left(S_{\bar{\tau}_{B}}=x\right) & =\sum_{k=1}^{\infty} P_{x}\left(S_{k}=z, \tau_{B \cup L_{0}}>k\right) \\
& =E_{x}\left[\text { number of visits to } z \text { in time interval }\left[0, \tau_{B \cup L_{0}}\right)\right] .
\end{aligned}
$$

Then taking the summation over all $z \in L_{N} \backslash B$, we have

$$
H_{B, N}(x)=E_{x}\left[\text { number of visits to } L_{N} \text { in time interval }\left[0, \tau_{B \cup L_{0}}\right)\right] \text {. }
$$

Then noting that $\tau_{L_{0}} \geq \tau_{B \cup L_{0}}$, we have

$$
H_{B, N}(x) \leq E_{x}\left[\text { number of visits to } L_{N} \text { in time interval }\left[0, \tau_{L_{0}}\right]\right] .
$$

Moreover, for $N>x_{2}$, note that if we trace the jumps on the second coordinate of $S_{n}$, it gives an (embedded) 1-dimensional simple random walk. We can use the strong Markov property of random walk on stopping time $\bar{\tau}_{L_{N}} \wedge \tau_{L_{0}}$

$E_{x}\left[\right.$ number of visits to $L_{N}$ in time interval $\left.\left[0, \tau_{L_{0}}\right]\right]$

$$
=\sum_{w \in L_{N}} P_{x}\left(\tau_{L_{N}}<\tau_{L_{0}}, S_{\tau_{L_{N}}}=w\right) E_{w}\left[\text { number of visits to } L_{N} \text { in time interval }\left[0, \tau_{L_{0}}\right]\right] \text {. }
$$


Note that for each $w \in L_{N}$,

(16)

$E_{w}\left[\right.$ number of visits to $L_{N}$ in time interval $\left.\left[0, \tau_{L_{0}}\right]\right]=\frac{4}{P_{w-(0,1)}\left(\tau_{L_{0}}<\tau_{L_{N}}\right)}=4 N$ is actually independent to the choice of $w$, and that for all $N>x_{2}$

$$
P_{x}\left(\tau_{L_{N}}<\tau_{L_{0}}\right)=\frac{x_{2}}{N}
$$

We have

$E_{x}\left[\right.$ number of visits to $L_{N}$ in time interval $\left.\left[0, \tau_{L_{0}}\right]\right]=4 N \cdot P_{x}\left(\tau_{L_{N}}<\tau_{L_{0}}\right)=4 x_{2}$.

Thus we have shown that

$$
H_{B, N}(x \rightarrow y) \leq H_{B, N}(x) \leq 4 x_{2}<\infty .
$$

Similarly, we can also show that for $x_{2}=0$,

$$
H_{B, N}(x \rightarrow y) \leq H_{B, N}(x) \leq 1<\infty .
$$

With $H_{B, N}(x \rightarrow y)$ uniformly bounded for all $N$, we next show that $H_{B, N}(x \rightarrow y)$ is monotonically decreasing with respect to $N$. I.e., for any $N>M>x_{2}+1$ we want to show that

$$
H_{B, N}(x \rightarrow y) \leq H_{B, M}(x \rightarrow y)
$$

Recalling that

$$
H_{B, N}(x \rightarrow y)=\sum_{z \in L_{N} \backslash B} P_{z}\left(S_{\bar{\tau}_{B \cup L_{0}}}=x, S_{\bar{\tau}_{B \cup L_{0}}-1}=y\right),
$$

for each $N$ we can define $S_{n}^{(0, N)}$ be a simple random walk in some probability space $P(\cdot)$ starting at $(0, N)$, and $S_{n}^{(k, N)}=S_{n}^{(0, N)}+(k, 0)$ for all $k \in \mathbb{Z}$. Noting that $S_{n}^{(k, N)}$ is a simple random walk starting at $(k, N)$, we have

$$
H_{B, N}(x)=\sum_{k:(k, N) \in L_{N} \backslash B} P\left(S_{\bar{\tau}_{B \cup L_{0}}}^{(k, N)}=x, S_{\bar{\tau}_{B \cup L_{0}}-1}^{(k, N)}=y\right) .
$$

Recalling that $N>M>x_{2}$, a random walk starting at $L_{N}$ must first visit $L_{M}$ before it can ever reach $x$. Thus for stopping time

$$
\bar{\tau}_{L_{M}}=\inf \left\{n: S_{n}^{(0, N)} \in L_{M}\right\}
$$

note that by definition we also have

$$
\bar{\tau}_{L_{M}}=\inf \left\{n: S_{n}^{(k, N)} \in L_{M}\right\}
$$

and

$$
S_{\bar{\tau}_{L_{M}}}^{(k, N)}=\underset{7}{k}+S_{\overline{\bar{\tau}}_{L_{M}}}^{(0, N)}
$$


for all $k \in \mathbb{Z}$. Thus by strong Markov property, we have for each $k$ such that $(k, N) \in L_{N} \backslash B$

$$
\begin{aligned}
& P\left(S_{\bar{\tau}_{B \cup L_{0}}}^{(k, N)}=x, S_{\bar{\tau}_{B \cup L_{0}}-1}^{(k, N)}=y\right) \\
& =\sum_{j \in Z} P\left(S_{\bar{\tau}_{L_{M}}}^{0, N}=(j, M), \bar{\tau}_{L_{M}} \leq \bar{\tau}_{B-(k, 0)}\right) P_{(j+k, M)}\left(S_{\bar{\tau}_{B \cup L_{0}}}=x, S_{\bar{\tau}_{B \cup L_{0}}-1}=y\right) \\
& \leq \sum_{j \in Z} P\left(S_{\bar{\tau}_{L_{M}}}^{0, N}=(j, M)\right) P_{(j+k, M)}\left(S_{\bar{\tau}_{B \cup L_{0}}}=x, S_{\bar{\tau}_{B \cup L_{0}}-1}=y\right) .
\end{aligned}
$$

Taking summation over all $k$,

$$
H_{B, N}(x) \leq \sum_{j \in \mathbb{Z}} P\left(S_{\bar{\tau}_{L_{M}}}^{(0, N)}=(j, M)\right) \sum_{k:(k, N) \in L_{N} \backslash B} P_{(j+k, M)}\left(S_{\bar{\tau}_{B \cup L_{0}}}=x, S_{\bar{\tau}_{B \cup L_{0}}-1}=y\right) .
$$

Note that for any $(i, M) \in B, P_{(i, M)}\left(S_{\bar{\tau}_{B \cup L_{0}}}=x\right)=0$. Thus

$$
\begin{aligned}
& \sum_{k:(k, N) \in L_{N} \backslash B} P_{(j+k, M)}\left(S_{\bar{\tau}_{B \cup L_{0}}}=x, S_{\bar{\tau}_{B \cup L_{0}}-1}=y\right) \\
\leq & \sum_{k:(k, M) \in L_{M} \backslash B} P_{(k, M)}\left(S_{\bar{\tau}_{B \cup L_{0}}}=x, S_{\bar{\tau}_{B \cup L_{0}}-1}=y\right)=H_{B, M}(x \rightarrow y) .
\end{aligned}
$$

Combining (21) and (22) we have (19). The fact that any monotonically decreasing nonnegative sequence is convergent finishes the proof of Proposition 1.

2.2. Proof of Proposition 2. To show Proposition 2 for finite subsets, recalling the definition and the fact that both $B_{1}$ and $B_{2}$ are finite. for any sufficiently large $N$ such that $L_{N} \cap B_{2}=\varnothing$, we have

$$
H_{B_{1}}=\sum_{x \in B_{1}} \sum_{z \in L_{N}} P_{z}\left(S_{\bar{\tau}_{B_{1} \cup L_{0}}}=x\right)
$$

and

$$
H_{B_{2}}=\sum_{x \in B_{2}} \sum_{z \in L_{N}} P_{z}\left(S_{\bar{\tau}_{B_{2} \cup L_{0}}}=x\right) .
$$

Changing the order of both summations we have

$$
H_{B_{1}}=\sum_{z \in L_{N}} \sum_{x \in B_{1}} P_{z}\left(S_{\bar{\tau}_{B_{1} \cup L_{0}}}=x\right)=\sum_{z \in L_{N}} P_{z}\left(S_{\bar{\tau}_{B_{1} \cup L_{0}}} \in B_{1}\right)=\sum_{z \in L_{N}} P_{z}\left(\tau_{B_{1}} \leq \tau_{L_{0}}\right)
$$

which is smaller than or equal to

$$
H_{B_{2}}=\sum_{z \in L_{N}} \sum_{x \in B_{2}} P_{z}\left(S_{\bar{\tau}_{B_{2}} \cup L_{0}}=x\right)=\sum_{z \in L_{N}} P_{z}\left(S_{\bar{\tau}_{B_{2}} \cup L_{0}} \in B_{2}\right)=\sum_{z \in L_{N}} P_{z}\left(\tau_{B_{2}} \leq \tau_{L_{0}}\right)
$$

by $(1)$. 


\section{UNIFORM UPPER BOUNDS ON HARMONIC MEASURE}

In this section, we improve the linear bound in (17) to Theorem 1 . Without loss of generality we can assume $x_{2}=n$. According to the definition of $H_{B, N}(x)$ and (2), we first note that for any $B^{\prime} \subset B$, with $x \in B^{\prime}$ and $L_{0} \subset B^{\prime}$,

$$
H_{B, N}(x) \leq H_{B^{\prime}, N}(x) \text {. }
$$

And since $B$ is connected and $L_{0} \subset B$. There must be a finite nearest neighbor path

$$
\mathcal{P}_{n}=\left\{x=P_{0}, P_{1}, P_{2}, \cdots, P_{k_{n}} \in L_{0}\right\}
$$

connecting $x$ and $L_{0}$, where $\left|P_{i}-P_{i+1}\right|=1$. And since $d\left(x, L_{0}\right)=n,\left|x-P_{k_{n}}\right| \geq n$. Define

$$
m_{n}=\inf \left\{i:\left|P_{i}-x\right| \geq n\right\}
$$

and

$$
\mathcal{Q}_{n}=\left\{P_{0}, P_{1}, P_{2}, \cdots, P_{m_{n}}\right\} .
$$

One can immediately see that

$$
\mathcal{Q}_{n} \subset B(x, 2 n) .
$$

Then for $B_{n}=L_{0} \cup \mathcal{Q}_{n}$, to prove Theorem 1, it suffices to show that

$$
H_{B_{n}, N}(x) \leq C n^{1 / 2} \text {. }
$$

And since simple random walk is translation invariant, we can without loss of generality assume that $x_{1}=0$. To show (23), we first prove that the inequalities in Lemma 3 and 4 and Inequality (2.15) in [7] are actually asymptotically sharp.

3.1. Asymptotic sharpness lemmas. In this subsection, we will temporally move back to $\mathbb{Z}^{2}$ rather than the upper half plane $\mathcal{H}$. The connection will be shown when we conclude the proof of Theorem 2. The "inverses" of both lemmas starts with similar arguments as in their original proof. While the inverse of Lemma 3 is on a more "natural" direction and its proof is more or less the same, that of Lemma 4 is a more delicate and requires a slightly stronger condition. Once we have the two inverse lemmas, the asymptotic sharpness of (2.15) follows from the decomposition of harmonic measure in [7].

Before the asymptotic sharpness results can be shown, we first introduce the discrete Green function used in [7] and quote some of its basic properties.

Lemma 3.1. (Lemma 1 of [7]) The series

$$
a(x)=\sum_{n=0}^{\infty}\left[P_{0}\left(S_{n}=0\right)-P_{n}\left(S_{n}=x\right)\right]
$$

converge for each $x \in \mathbb{Z}^{2}$, and the function a $(\cdot)$ has the following properties:

$$
a(x) \geq 0, \forall x \in \mathbb{Z}^{2}, a(0)=0,
$$




$$
\begin{aligned}
& a(( \pm 1,0))=a((0, \pm 1))=1 \\
& E_{x}\left[a\left(S_{1}\right)\right]-a(x)=\delta(x, 0),
\end{aligned}
$$

so $a\left(S_{n \wedge \tau_{v}}-v\right)$ is a nonnegative martingale, where $\tau_{v}=\tau_{\{v\}}$, for any $v \in \mathbb{Z}^{2}$. And there is some suitable $C_{0}, C_{1}<\infty$ such that for all $x \neq 0$,

$$
x^{2}\left|a(x)-\frac{1}{2 \pi} \log \right| x\left|-C_{0}\right| \leq C_{1} .
$$

Here for any positive $R$ we use the notation

$$
\tau_{R}=\tau_{\partial^{\text {out }} B(0, R)} \cdot
$$

We have

Lemma 3.2. (Inverse of Lemma 3 of [7]) Let $D \subset\{u:|u| \leq r\}$ contain the origin. Then for all $R$ sufficiently larger than $r$ one has uniformly in $v \in \partial^{\text {out }} B(0, R)$ and in $D$

$$
P_{v}\left(\tau_{D}<\tau_{R}\right) \leq C[R \log (R)]^{-1} .
$$

Proof. For any $v \in \partial^{\text {out }} B(0, R)$, there must be at least one point among its 4 neighbors within $B(0, R)$. And for each such point $w$, we use the same martingale

$$
Y_{n}=a\left(S_{n \wedge \tau_{0}}\right)
$$

as the Lemma 3 of [7], with $S_{0}=w$. Then since $0 \in D$, we have that the stopping time $\sigma=\tau_{D} \wedge \tau_{R} \leq \tau_{0}$, and

$$
\begin{aligned}
a(w) & =E_{w}\left[a\left(S_{\sigma}\right) \mathbb{1}_{\tau_{D}<\tau_{R}}\right]+E_{w}\left[a\left(S_{\sigma}\right) \mathbb{1}_{\tau_{R}<\tau_{D}}\right] \\
& =P_{w}\left(\tau_{D}<\tau_{R}\right) E_{w}\left[a\left(S_{\sigma}\right) \mid \tau_{D}<\tau_{R}\right]+P_{w}\left(\tau_{R}<\tau_{D}\right) E_{w}\left[a\left(S_{\sigma}\right) \mid \tau_{R}<\tau_{D}\right] \\
& =E_{w}\left[a\left(S_{\sigma}\right) \mid \tau_{R}<\tau_{D}\right]-P_{w}\left(\tau_{D}<\tau_{R}\right)\left(E_{w}\left[a\left(S_{\sigma}\right) \mid \tau_{R}<\tau_{D}\right]-E_{w}\left[a\left(S_{\sigma}\right) \mid \tau_{D}<\tau_{R}\right]\right) .
\end{aligned}
$$

Thus we have

$$
P_{w}\left(\tau_{D}<\tau_{R}\right)=\frac{E_{w}\left[a\left(S_{\sigma}\right) \mid \tau_{R}<\tau_{D}\right]-a(w)}{E_{w}\left[a\left(S_{\sigma}\right) \mid \tau_{R}<\tau_{D}\right]-E_{w}\left[a\left(S_{\sigma}\right) \mid \tau_{D}<\tau_{R}\right]} .
$$

Note that under $\left\{\tau_{R}<\tau_{D}\right\}, S_{\sigma} \in \partial^{\text {out }} B(0, R)$, which implies $R \leq\left|S_{\sigma}\right| \leq R+1$.

We have by (28) for sufficiently large $R$

$$
\begin{aligned}
E_{w}\left[a\left(S_{\sigma}\right) \mid \tau_{R}<\tau_{D}\right] & \leq E\left[\frac{1}{2 \pi} \log \left(\left|S_{\sigma}\right|\right)+C_{0}+\frac{C_{1}}{\left|S_{\sigma}\right|^{2}} \mid \tau_{R}<\tau_{D}\right] \\
& \leq \frac{1}{2 \pi} \log (R+1)+C_{0}+\frac{C_{1}}{R^{2}} \\
& \leq \frac{1}{2 \pi} \log (R)+\frac{1}{2 \pi R}+C_{0}+\frac{C_{1}}{R^{2}} .
\end{aligned}
$$


The last inequality is from the fact that $\log (1+x) \leq x$. On the other hand, we can also have

$$
\begin{aligned}
E_{w}\left[a\left(S_{\sigma}\right) \mid \tau_{R}<\tau_{D}\right] & \geq E\left[\frac{1}{2 \pi} \log \left(\left|S_{\sigma}\right|\right)+C_{0}-\frac{C_{1}}{\left|S_{\sigma}\right|^{2}} \mid \tau_{R}<\tau_{D}\right] \\
& \geq \frac{1}{2 \pi} \log (R)+C_{0}-\frac{C_{1}}{R^{2}}
\end{aligned}
$$

Similarly, note that $R-1 \leq|w| \leq R$, we have sufficiently large $R$

$$
\begin{aligned}
a(w) & \geq \frac{1}{2 \pi} \log \left(\left|a_{w}\right|\right)+C_{0}-\frac{C_{1}}{\left|a_{w}\right|^{2}} \\
& \geq \frac{1}{2 \pi} \log (R-1)+C_{0}-\frac{2 C_{1}}{R^{2}} \\
& \geq \frac{1}{2 \pi} \log (R)-\frac{1}{\pi R}+C_{0}-\frac{2 C_{1}}{R^{2}} .
\end{aligned}
$$

Now the last inequality is from the fact that $\log \left(1-R^{-1}\right)=-R^{-1}+O\left(-R^{-2}\right)>$ $-2 R^{-1}$ for sufficiently large $R$. Finally, we can use the same argument and have for sufficiently large $R$

$$
\begin{aligned}
E_{w}\left[a\left(S_{\sigma}\right) \mid \tau_{D}<\tau_{R}\right] & =E_{w}\left[a\left(S_{\sigma}\right) \mathbb{1}_{S_{\sigma} \neq 0} \mid \tau_{D}<\tau_{R}\right] \\
& \leq \frac{1}{2 \pi} \log (r)+C_{0}+C_{1} \\
& \leq \frac{1}{4 \pi} \log (R)+C_{0}-\frac{C_{1}}{R^{2}} .
\end{aligned}
$$

From (30) one immediately has that

$$
P_{w}\left(\tau_{D}<\tau_{R}\right) \leq \frac{\bar{E}_{w}\left[a\left(S_{\sigma}\right) \mid \tau_{R}<\tau_{D}\right]-\underline{a}(w)}{\underline{E}_{w}\left[a\left(S_{\sigma}\right) \mid \tau_{R}<\tau_{D}\right]-\bar{E}_{w}\left[a\left(S_{\sigma}\right) \mid \tau_{D}<\tau_{R}\right]},
$$

where $\overline{(\cdot)}$ stands for an upper bound while $(\cdot)$ for a lower bound. Then substitute (31)-(34) into (35), we have

$$
P_{w}\left(\tau_{D}<\tau_{R}\right) \leq \frac{\frac{3}{2 \pi R}+\frac{3 C_{1}}{R^{2}}}{\frac{1}{4 \pi} \log (R)} \leq 7[R \log R]^{-1}
$$

for sufficiently large $R$. Finally note that

$$
P_{v}\left(\tau_{D}<\tau_{R}\right) \leq \sup _{w \in B(0, R):|w-v|=1} P_{w}\left(\tau_{D}<\tau_{R}\right) .
$$

Thus the proof of this lemma is complete.

Our next lemma gives an "inverse" for Lemma 4 of of [7], under a slightly stronger condition. 
Lemma 3.3. (Inverse of Lemma 4 of [7]) There are constants $3<C_{2}<\infty$ and $c_{2}>0$ such that for all $r$ and $R$ sufficiently larger than $r$, any $D \subset\{u:|u| \leq r\}$, and for any $z \in \partial^{\text {out }} B\left(0, C_{2} \cdot r\right)$, we have

$$
P_{z}\left(\tau_{R}<\tau_{D}\right) \geq \frac{c_{2}}{\log (R)}
$$

Proof. Again, we first consider the same martingale as in the original lemma. For each $z \in \partial^{\text {out }} B\left(0, C_{2} \cdot r\right)$ define

$$
Z_{n}=\sum_{v \in D} a\left(S_{n \wedge \tau_{D}}-v\right)
$$

with $S_{0}=z$. Recall that $\sigma=\tau_{D} \wedge \tau_{R} \leq \tau_{D}$. Using the same argument as in Lemma 4 of [7] and Lemma 3.2 above, we have

$$
\begin{aligned}
\sum_{v \in D} a(z-v) & =P_{z}\left(\tau_{R}<\tau_{D}\right)\left(\sum_{v \in D} E\left[a\left(S_{\sigma}-v\right) \mid \tau_{R}<\tau_{D}\right]\right) \\
& +\left[1-P_{z}\left(\tau_{R}<\tau_{D}\right)\right]\left(\sum_{v \in D} E\left[a\left(S_{\sigma}-v\right) \mid \tau_{D}<\tau_{R}\right]\right),
\end{aligned}
$$

which gives us

$$
\begin{aligned}
P_{z}\left(\tau_{R}<\tau_{D}\right) & =\frac{\sum_{v \in D} a(z-v)-\sum_{v \in D} E\left[a\left(S_{\sigma}-v\right) \mid \tau_{D}<\tau_{R}\right]}{\sum_{v \in D} E\left[a\left(S_{\sigma}-v\right) \mid \tau_{R}<\tau_{D}\right]-\sum_{v \in D} E\left[a\left(S_{\sigma}-v\right) \mid \tau_{R}<\tau_{D}\right]} \\
& \geq \frac{\sum_{v \in D} a(z-v)-\sum_{v \in D} E\left[a\left(S_{\sigma}-v\right) \mid \tau_{D}<\tau_{R}\right]}{\sum_{v \in D} E\left[a\left(S_{\sigma}-v\right) \mid \tau_{R}<\tau_{D}\right]} \\
& \geq \frac{\sum_{v \in D} a(z-v)-\bar{\sum}_{v \in D} E\left[a\left(S_{\sigma}-v\right) \mid \tau_{D}<\tau_{R}\right]}{\sum_{v \in D} E\left[a\left(S_{\sigma}-v\right) \mid \tau_{R}<\tau_{D}\right]}
\end{aligned}
$$

where $\overline{(\cdot)}$ again stands for an upper bound while $(\cdot)$ for a lower bound. Then for any $z \in \partial^{\text {out }} B\left(0, C_{2} \cdot r\right)$ and any $v \in D \subset B(0, \bar{r}),|z-v| \geq\left(C_{2}-1\right) r$, which implies

$$
\begin{aligned}
a(z-v) & \geq \frac{1}{2 \pi} \log (|z-v|)+C_{0}-\frac{C_{1}}{|z-v|^{2}} \\
& \geq \frac{1}{2 \pi} \log (r)+\frac{\log \left(C_{2}-1\right)}{2 \pi}+C_{0}-C_{1} .
\end{aligned}
$$

Taking summation over all $v \in D$, we have

$$
\sum_{v \in D} a(z-v) \geq \frac{|D|}{2 \pi} \log (r)+\left[\frac{\log \left(C_{2}-1\right)}{2 \pi}+C_{0}-C_{1}\right] \cdot|D| .
$$


Then under $\left\{\tau_{D}<\tau_{R}\right\}, S_{\sigma} \in D$, which implies that $\left|S_{\sigma}-v\right| \leq 2 r$ for all $v \in D \subset$ $B(0, r)$. Then we have

$$
\begin{aligned}
E\left[a\left(S_{\sigma}-v\right) \mid \tau_{D}<\tau_{R}\right] & =E\left[a\left(S_{\sigma}-v\right) \mathbb{1}_{S_{\sigma} \neq v} \mid \tau_{D}<\tau_{R}\right] \\
& \leq E\left[\left(\frac{1}{2 \pi} \log \left(\left|S_{\sigma}-v\right|\right)+C_{0}+\frac{C_{1}}{\left|S_{\sigma}-v\right|^{2}}\right) \mathbb{1}_{S_{\sigma} \neq v} \mid \tau_{D}<\tau_{R}\right] \\
& \leq \frac{1}{2 \pi} \log (r)+\left[\frac{\log (2)}{2 \pi}+C_{0}+C_{1}\right] .
\end{aligned}
$$

Again taking summation over all $v \in D$, we have

$$
\sum_{v \in D} E\left[a\left(S_{\sigma}-v\right) \mid \tau_{D}<\tau_{R}\right] \leq \frac{|D|}{2 \pi} \log (r)+\left[\frac{\log (2)}{2 \pi}+C_{0}+C_{1}\right] \cdot|D| .
$$

Finally, under $\left\{\tau_{R}<\tau_{D}\right\}, S_{\sigma} \in \partial^{\text {out }} B(0, R)$, so for any $v \in D, R-r \leq\left|S_{\sigma}-v\right| \leq$ $R+r$. Thus

$$
E\left[a\left(S_{\sigma}-v\right) \mid \tau_{R}<\tau_{D}\right] \leq \frac{1}{2 \pi} \log (R+r)+C_{0}+\frac{C_{1}}{(R-r)^{2}} \leq \frac{1}{\pi} \log (R)
$$

for all $R$ sufficiently larger than $r$. Thus we have

$$
\sum_{v \in D} E\left[a\left(S_{\sigma}-v\right) \mid \tau_{R}<\tau_{D}\right] \leq \frac{|D|}{\pi} \log (R) .
$$

Now, we can substitute (39)-(41) into (38),

$$
P_{z}\left(\tau_{R}<\tau_{D}\right) \geq \frac{\left[\frac{\log \left(C_{2}-1\right)}{2 \pi}-\frac{\log (2)}{2 \pi}-2 C_{1}\right] \cdot|D|}{\frac{|D|}{\pi} \log (R)}=\frac{\log \left(\frac{C_{2}-1}{2}\right)-4 \pi C_{1}}{2 \log (R)},
$$

and let $C_{2}=2 \exp \left(4 \pi C_{1}+1\right)+1$, and $c_{2}=1 / 2$ to complete the proof.

With Lemma 3.2 and Lemma 3.3, we are now able to show the asymptotic sharpness of (2.15) in [7] and have

Lemma 3.4. There is a constant $c_{3}>0$ such that for all $D \subset\{u:|u| \leq r\}$ contains the origin, and any $y \in D$

$$
\mu_{D}(y) \geq c_{3} P_{y}\left(\tau_{C_{2} \cdot r}<\tau_{D}\right) .
$$

Here $\mu_{D}(\cdot)$ is the harmonic measure on $\mathbb{Z}^{2}$ associated with $D$,

$$
\mu_{D}(y)=\lim _{|z| \rightarrow \infty} P_{z}\left(S_{\tau_{D}}=y\right) .
$$

Proof. Here we follow exactly the same decomposition according to first hitting position, as in [7]. There is a $c>0$ such that for all sufficiently large $R$,

$$
\mu_{D}(y) \geq \frac{c}{R} \sum_{z \in \partial^{\text {out }} B(0, R)} P_{z}\left(S_{\tau_{D}}=y\right) .
$$


And for any $z \in \partial^{\text {out }} B(0, R)$, we again have

$$
\begin{aligned}
P_{z}\left(S_{\tau_{D}}=y\right) & =\sum_{n=1}^{\infty} P_{z}\left(\tau_{D}=n, S_{n}=y\right) \\
& =\sum_{n=1}^{\infty} P_{y}\left(\tau_{D}>n, S_{n}=z\right) \\
& =E_{y}\left[\# \text { of visits to } z \text { in }\left[0, \tau_{D}\right]\right] .
\end{aligned}
$$

Thus we have

$$
\mu_{D}(y) \geq \frac{c}{R} E_{y}\left[\# \text { of visits to } \partial^{\text {out }} B(0, R) \text { in }\left[0, \tau_{D}\right]\right] .
$$

Apply strong Markov property on the expectation, we have

$$
\begin{aligned}
E_{y} & {\left[\# \text { of visits to } \partial^{\text {out }} B(0, R) \text { in }\left[0, \tau_{D}\right]\right] } \\
& =\sum_{w \in \partial^{\text {out }} B(0, R)} P_{y}\left(\tau_{R}<\tau_{D}, S_{\tau_{R}}=w\right) E_{w}\left[\# \text { of visits to } \partial^{\text {out }} B(0, R) \text { in }\left[0, \tau_{D}\right]\right] .
\end{aligned}
$$

Then for each $w \in \partial^{\text {out }} B(0, R)$,

$$
\begin{aligned}
E_{w} & {\left[\# \text { of visits to } \partial^{\text {out }} B(0, R) \text { in }\left[0, \tau_{D}\right]\right] } \\
& =\sum_{k=0}^{\infty} P_{w}\left(S \text { returns at least } k \text { times to } \partial^{\text {out }} B(0, R) \text { before } \tau_{D}\right) \\
& \geq \sum_{k=0}^{\infty}\left(\inf _{v \in \partial^{\text {out }} B(0, R)}\left\{P_{v}\left(\tau_{R}<\tau_{D}\right)\right\}\right)^{k} \\
& =\frac{1}{1-\inf _{v \in \partial^{\text {out }} B(0, R)}\left\{P_{v}\left(\tau_{R}<\tau_{D}\right)\right\}} \\
& =\frac{1}{\sup _{v \in \partial^{\text {out }} B(0, R)}\left\{P_{v}\left(\tau_{D}<\tau_{R}\right)\right\}} .
\end{aligned}
$$

Note that in Lemma 3.2 we proved that

$$
\sup _{v \in \partial^{\text {out }} B(0, R)}\left\{P_{v}\left(\tau_{D}<\tau_{R}\right)\right\} \leq C[R \log (R)]^{-1} .
$$

Plug it to (45), we have for all $w \in \partial^{\text {out }} B(0, R)$,

$$
E_{w}\left[\# \text { of visits to } \partial^{\text {out }} B(0, R) \text { in }\left[0, \tau_{D}\right]\right] \geq \frac{1}{C} R \log (R) .
$$


Then combining (43), (44) and (46),

$$
\begin{aligned}
\mu_{D}(y) & \geq \frac{c}{R} \cdot \frac{1}{C} R \log (R) \sum_{w \in \partial^{\text {out }} B(0, R)} P_{y}\left(\tau_{R}<\tau_{D}, S_{\tau_{R}}=w\right) \\
& =\frac{c}{C} \log (R) P_{y}\left(\tau_{R}<\tau_{D}\right) .
\end{aligned}
$$

Then for $P_{y}\left(\tau_{R}<\tau_{D}\right)$, note that for sufficiently large $R>C_{2} \cdot r$, if a random walk wants to exit $B(0, R)$, it has to exit $B\left(0, C_{2} \cdot r\right)$ first. Thus, again by strong Markov property, we have

$$
P_{y}\left(\tau_{R}<\tau_{D}\right)=\sum_{z \in \partial^{\text {out }} B\left(0, C_{2} \cdot r\right)} P_{y}\left(\tau_{C_{2} \cdot r}<\tau_{D}, S_{\tau_{C_{2} \cdot r}}=z\right) P_{z}\left(\tau_{R}<\tau_{D}\right) .
$$

Since in Lemma 3.3, we prove that for any $z \in \partial^{\text {out }} B\left(0, C_{2} \cdot r\right)$,

$$
P_{z}\left(\tau_{R}<\tau_{D}\right) \geq \frac{c_{2}}{\log (R)}
$$

we have

$$
\begin{aligned}
P_{y}\left(\tau_{R}<\tau_{D}\right) & \geq \frac{c_{2}}{\log (R)} \sum_{z \in \partial^{\text {out }} B\left(0, C_{2} \cdot r\right)} P_{y}\left(\tau_{C_{2} \cdot r}<\tau_{D}, S_{\tau_{C_{2} \cdot r}}=z\right) \\
& =\frac{c_{2}}{\log (R)} P_{y}\left(\tau_{C_{2} \cdot r}<\tau_{D}\right) .
\end{aligned}
$$

Combining (47) and (48), and let $c_{3}=c \cdot c_{2} / C$, the proof of Lemma 3.4 is complete.

3.2. Proof of Theorem 1. Now we have the tools we need to finish the proof of Theorem 1. Recall that $B_{n}=L_{0} \cup \mathcal{Q}_{n}$, and that by (15), strong Markov property, and (16)

(49)

$$
\begin{aligned}
& H_{B_{n}, N}\left(y_{n}\right) \\
& =E_{y_{n}}\left[\text { number of visits to } L_{N} \text { in time interval }\left[0, \tau_{B_{n}}\right]\right] \\
& =\sum_{w \in L_{N}} P_{y_{n}}\left(\tau_{L_{N}}<\tau_{B_{n}}, S_{\tau_{L_{N}}}=w\right) E_{w}\left[\text { number of visits to } L_{N} \text { in time interval }\left[0, \tau_{B_{n}}\right]\right] \\
& \leq \sum_{w \in L_{N}} P_{y_{n}}\left(\tau_{L_{N}}<\tau_{B_{n}}, S_{\tau_{L_{N}}}=w\right) E_{w}\left[\text { number of visits to } L_{N} \text { in time interval }\left[0, \tau_{L_{0}}\right]\right] \\
& =4 N \cdot P_{y_{n}}\left(\tau_{L_{N}}<\tau_{B_{n}}\right) .
\end{aligned}
$$

So in order to show (23) and thus Theorem 1, it is sufficient to prove that

$$
P_{y_{n}}\left(\tau_{L_{N}}<\tau_{B_{n}}\right) \leq \frac{C n^{1 / 2}}{N} \text {. }
$$


To show (50), define $r_{n}=2 n, \mathcal{S}_{n}=\partial^{\text {out }} B\left(y_{n}, C_{2} \cdot r_{n}\right) \cap\left\{(x, y) \in \mathbb{Z}^{2}, y \geq 1\right\}$. Note that if a simple random walk starting at $y_{n}$ wants to reach $L_{N}$ before returning to $B_{n}$, it has to visit some point in $\mathcal{S}_{n}$ first. Thus once again by strong Markov property,

$$
P_{y_{n}}\left(\tau_{L_{N}}<\tau_{B_{n}}\right)=\sum_{z \in \mathcal{S}_{n}} P_{y_{n}}\left(\tau_{\mathcal{S}_{n}}<\tau_{B_{n}}, S_{\tau_{\mathcal{S}_{n}}}=z\right) P_{z}\left(\bar{\tau}_{L_{N}}<\bar{\tau}_{B_{n}}\right) .
$$

Note that for each $z \in \mathcal{S}_{n}$, by (2) and the fact that $L_{0} \subset B_{n}$,

$$
P_{z}\left(\bar{\tau}_{L_{N}}<\bar{\tau}_{B_{n}}\right) \leq P_{z}\left(\bar{\tau}_{L_{N}}<\bar{\tau}_{L_{0}}\right) \leq \frac{\left(2 C_{2}+1\right) n}{N} .
$$

Plugging this uniform upper bound into (51), we now have

$$
P_{y_{n}}\left(\tau_{L_{N}}<\tau_{B_{n}}\right) \leq P_{y_{n}}\left(\tau_{\mathcal{S}_{n}}<\tau_{B_{n}}\right) \cdot \frac{\left(2 C_{2}+1\right) n}{N} .
$$

Thus for Theorem 1 it is sufficient to show that

$$
P_{y_{n}}\left(\tau_{\mathcal{S}_{n}}<\tau_{B_{n}}\right) \leq C n^{-1 / 2} .
$$

Noting that $\mathcal{S}_{n} \subset \partial^{\text {out }} B\left(y_{n}, C_{2} \cdot r_{n}\right)$, and that $\mathcal{Q}_{n} \subset B_{n}$, then by (1) and (2),

$$
P_{y_{n}}\left(\tau_{\mathcal{S}_{n}}<\tau_{B_{n}}\right) \leq P_{y_{n}}\left(\tau_{\partial^{\text {out }} B\left(y_{n}, C_{2} \cdot r_{n}\right)}<\tau_{\mathcal{Q}_{n}}\right) .
$$

Since simple random walk is translation invariant,

$$
P_{y_{n}}\left(\tau_{\partial^{\text {out }} B\left(y_{n}, C_{2} \cdot r_{n}\right)}<\tau_{\mathcal{Q}_{n}}\right)=P_{0}\left(\tau_{C_{2} \cdot r_{n}}<\tau_{D_{n}}\right),
$$

where $D_{n}=\mathcal{Q}_{n}-y_{n}$, which is a connected subset of $B\left(0, r_{n}\right)$ containing 0 . Then apply Lemma 3.4 on $y=0 \in D_{n} \subset B\left(0, r_{n}\right)$, we have

$$
P_{0}\left(\tau_{C_{2} \cdot r_{n}}<\tau_{D_{n}}\right) \leq \frac{1}{c_{3}} \mu_{D_{n}}(0) .
$$

Finally by Theorem 1 (the only theorem) of [7], and the fact that $0 \in D_{n}$, with $D_{n}$ connected and $r\left(D_{n}\right) \in[n, 2 n]$.

$$
\mu_{D_{n}}(0) \leq C n^{-1 / 2},
$$

which finishes the proof of Theorem 1.

\section{Subset maximizing the Stationary harmonic measure}

In this section we prove Theorem 2. Then together with the uniform upper bound we had in Theorem 1, one can see that $U_{n}=V_{n} \cup L_{0}$ is the subset maximizing harmonic measure up to multiplying a constant.

Before we start with the details, an outline of the proof of Theorem 2 is presented. See also Figure 1. The detailed proof will piece together everything we need in the list below, although the order that each lemma is proved may not be precisely consistent with the outline. 
(i) We have found that $H_{U_{n}, N}\left(y_{n}\right)$ equals to the expected number of visits to $L_{N}$ before a simple random walk $S$ starting from $y_{n}$ returns to $U_{n}$. If the random walk reaches $L_{N}$ first before returning to $U_{n}$, the expected number of (re-) visits is $4 N+o(N)$.

(ii) For $S$ to reach $L_{N}$ first before returning to $U_{n}$, it has to reach $L_{2 n}$ first. Once it reaches $L_{2 n}$, the probability of success from there is at least of oder $n / N$.

(iii) If $S$ reached the upper outer boundary of the $L_{1}$ ball $B_{1}\left(y_{n}, n / 3\right)=\{|x|+$ $|y-n| \leq n / 3\}$ before returning to $V_{n}$, by the invariance principle there is a positive probability for it to continue to $L_{2 n}$ before returning to $U_{n}$.

(iv) The probability that $S_{n}$ exits $B_{1}\left(y_{n}, n / 3\right)$ before returning to $V_{n}$ is at least $O\left(n^{-1 / 2}\right)$.

(v) Given $S_{n}$ exits $B_{1}\left(y_{n}, n / 3\right)$ before returning to $V_{n}$, it is more likely to exit from the upper half than the lower half.

Without loss of generality, we only need to prove this theorem for $n$ sufficiently large and $N$ sufficiently larger than $n$.

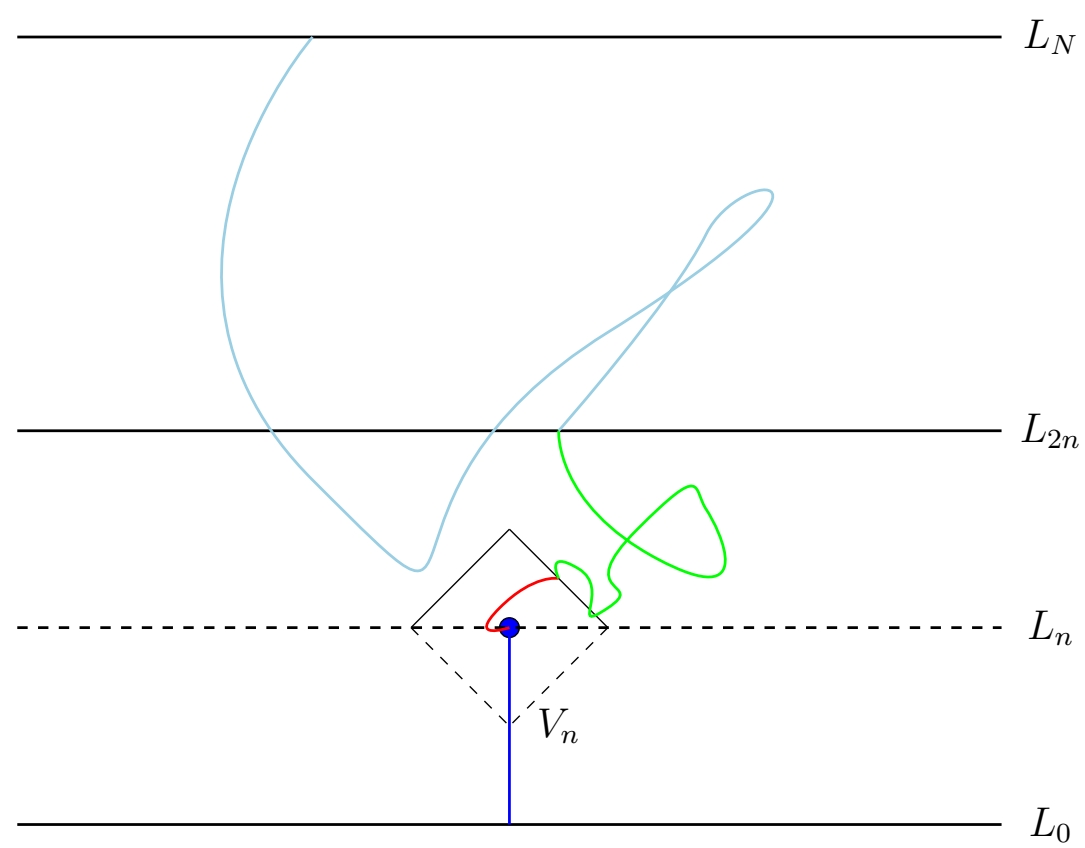

FiguRE 1. Outline for the lower bound

4.1. Lower bound on escaping probability. To show that $S_{n}$ exits $B_{1}\left(y_{n}, n / 3\right)$ before returning to $V_{n}$ with probability at least $O\left(n^{-1 / 2}\right)$, we need to prove one more asymptotic sharpness result which is basically an inverse of Lemma 6 in [7] with $y=0$. Here we first introduce the definition of the infinite range 1-dimensional random walk in their problem and quote its properties: 
Let $S_{n}, n \geq 0$ be a 2-dimensional simple random walk starting from the $y$ - (or equivalently $x-$ ) axis. Define the stopping times $\sigma_{0}=0$,

$$
\sigma_{k+1}=\inf \left\{n>\sigma_{k}, S_{n} \in y \text {-axis }\right\},
$$

and

$$
T_{k}=y \text {-coornidate of } S_{\sigma_{k}}, \quad Y_{k+1}=T_{k+1}-T_{k} .
$$

Note that now $T_{k}$ is a 1-dimensional random walk although each of its steps has no well defined expectation. Moreover, define $\rho$ to be a stopping time for $T$

$$
\rho=\inf \left\{k \geq 1, T_{k} \leq 0\right\} .
$$

We can also define the Green function for the random walk $T$ stopped at $\rho$ as

$$
G(j, l)=E_{j}[\text { number of visits by } T \text { to } l \text { before } \rho], \quad j, l>0 .
$$

The following properties of $G(\cdot, \cdot)$ have been proved

Lemma 4.1. (Lemma 5 in [7]) For $j, l>0$,

$$
G(j, l)=\sum_{n=1}^{j \wedge l} v(j-n) v(l-n)
$$

for some numbers $v(\cdot)$ satisfying

$$
\begin{aligned}
& v(n) \geq 0, \\
& V(n)=\sum_{i=0}^{n} v(k) \sim C \sqrt{n}, \\
& V\left(T_{n \wedge \rho}\right) \text { is a nonnegative martingale under } P_{j}, j>0 .
\end{aligned}
$$

Moreover, in Equation (2.27) of [7] it has been proved that

$$
P\left(Y_{1}<-n\right) \sim \frac{1}{2 n}
$$

and by symmetry

$$
P\left(Y_{1}>n\right) \sim \frac{1}{2 n}
$$

Now we have all the tools needed to get the following lemma:

Lemma 4.2. (Inverse of Lemma 6 in [7]) There is a constant $c>0$ such that for all $r \geq 1$, one has

$$
P_{0}\left(T_{\rho}<-r\right) \geq c r^{-1 / 2}
$$


Proof. Condition on the location of $T_{1}$, we have

$$
\begin{aligned}
P_{0}\left(T_{\rho}<-r\right) & =P_{0}\left(Y_{1}<-r\right)+\sum_{j=1}^{\infty} P\left(Y_{1}=j\right) P_{j}\left(T_{\rho}<-r\right) \\
& \geq \sum_{j=1}^{\infty} P\left(Y_{1}=j\right) P_{j}\left(T_{\rho}<-r\right) .
\end{aligned}
$$

For each $j>0$,

$$
P_{j}\left(T_{\rho}<-r\right)=\sum_{l=1}^{\infty} \sum_{n=1}^{\infty} P_{j}\left(\rho=n, T_{n-1}=l, T_{n}<-r\right),
$$

while for each $n, j, l \geq 0$,

$$
\begin{aligned}
P_{j}\left(\rho=n, T_{n-1}=l, T_{n}<-r\right) & =P_{j}\left(\rho>n-1, T_{n-1}=l, T_{n}<-r\right) \\
& =P_{j}\left(\rho>n-1, T_{n-1}=l\right) P_{l}\left(T_{1}<-r\right) \\
& =P_{j}\left(\rho>n-1, T_{n-1}=l\right) P\left(Y_{1}<-r-l\right) .
\end{aligned}
$$

Taking the summation we have

$$
P_{j}\left(T_{\rho}<-r\right)=\sum_{l=1}^{\infty} G(j, l) P\left(Y_{1}<-r-l\right)
$$

and thus by (60)-(62)

$$
\begin{aligned}
P_{0}\left(T_{\rho}<-r\right) & \geq \sum_{j=1}^{\infty} P\left(Y_{1}=j\right) \sum_{l=1}^{\infty} G(j, l) P\left(Y_{1}<-r-l\right) \\
& \geq c \sum_{j=1}^{\infty} P\left(Y_{1}=j\right) \sum_{l=1}^{\infty} G(j, l) \frac{1}{r+l} .
\end{aligned}
$$

Then by Lemma 4.1 we have

$$
\begin{aligned}
P_{0}\left(T_{\rho}<-r\right) & \geq c \sum_{j=1}^{\infty} P\left(Y_{1}=j\right) \sum_{l=1}^{\infty} G(j, l) \frac{1}{r+l} \\
& \geq c \sum_{j=1}^{\infty} P\left(Y_{1}=j\right) \sum_{l=1}^{\infty} \frac{1}{r+l} \sum_{n=1}^{j \wedge l} v(j-n) v(l-n) \\
& =c \sum_{j=1}^{\infty} P\left(Y_{1}=j\right) \sum_{n=1}^{j} v(j-n) \sum_{l=n}^{\infty} v(l-n) \frac{1}{r+l}
\end{aligned}
$$

Noting that for each $l \geq n$,

$$
\frac{1}{r+l}=\frac{1}{(r+l)(r+l+1)}+\frac{1}{(r+l+1)(r+l+2)}+\cdots,
$$


with summation by parts and Lemma 4.1 we have for each $n$

$$
\begin{aligned}
\sum_{l=n}^{\infty} v(l-n) \frac{1}{r+l} & =\sum_{m=0}^{\infty} \frac{V(m)}{(r+n+m)(r+n+m+1)} \\
& \geq c \sum_{m=0}^{\infty} \frac{\sqrt{m}}{(r+n+m)(r+n+m+1)} \\
& =c \sum_{m=1}^{\infty} \frac{\sqrt{m}-\sqrt{m-1}}{r+n+m} \\
& \geq \frac{1}{2} \sum_{m=1}^{\infty} \frac{1}{(r+n+m)^{3 / 2}} \\
& \geq \frac{1}{2} \int_{r+n+1}^{\infty} \frac{1}{x^{3 / 2}} \geq \frac{1}{2}(r+n)^{-1 / 2} .
\end{aligned}
$$

Then noting that for $l=j=1$,

$$
G(j, l)=\sum_{n=1}^{j \wedge l} v(j-n) v(l-n)=v(0)^{2},
$$

and that by definition $G(1,1) \geq 1>0$, we have $v(0) \geq 1$. Moreover, note that $P\left(Y_{1}=1\right) \geq P_{0}\left(S_{1}=(0,1)\right)=1 / 4$. Since all the terms in (64) are nonnegative, let $j=n=1$, we have

$$
\begin{aligned}
P_{0}\left(T_{\rho}<-r\right) & \geq c P\left(Y_{1}=1\right) v(0) \sum_{l=1}^{\infty} v(l-1) \frac{1}{r+l} \\
& \geq \frac{c}{8}(r+1)^{-1 / 2} \geq \frac{c}{16} r^{-1 / 2} .
\end{aligned}
$$

Thus the proof of Lemma 4.2 is complete.

With Lemma 4.2 the proof for the desired lower bound of escaping probability is straightforward. Recall that we have a 2-dimensional simple random walk starting at $y_{n}=(0, n)$. Define $V_{n}^{\prime}=\{(0, y), n-[n / 3] \leq y \leq n\}$ and

$$
\mathcal{S}_{1, n}=\partial B_{1}\left(y_{n},[n / 3]\right) \text {. }
$$

Here note that for $L_{1}$ ball $B_{1}\left(y_{n},[n / 3]\right)$ we do not need to specify if the boundary is in or out. Then for $C_{n}^{\prime}=\{(0, y), y<n-[n / 3]\}$, note that for a 2-dimensional simple random walk starting at $y_{n}=(0, n)$ we always have

$$
\tau_{\mathcal{S}_{1, n}}<\tau_{C_{n}^{\prime}}
$$

Thus for the escaping probability we want to bound from below, we have

$$
P_{y_{n}}\left(\tau_{\mathcal{S}_{1, n}}<\tau_{U_{n}}\right)=P_{y_{n}}\left(\tau_{\mathcal{S}_{1, n}}<\tau_{V_{n}^{\prime}}\right) \geq P_{y_{n}}\left(\tau_{C_{n}^{\prime}}<\tau_{V_{n}^{\prime}}\right) .
$$


By the translation invariance of $S$,

$$
P_{y_{n}}\left(\tau_{C_{n}^{\prime}}<\tau_{V_{n}^{\prime}}\right)=P_{0}\left(\tau_{C_{n}^{\prime}-y_{n}}<\tau_{V_{n}^{\prime}-y_{n}}\right) .
$$

Note that $C_{n}^{\prime}-y_{n}=\{(0, y), y<-[n / 3]\}$ and that $V_{n}^{\prime}-y_{n}=\{(0, y),-[n / 3] \leq$ $y \leq 0\}$. Let $r=[n / 3]$ one has

$$
P_{0}\left(\tau_{C_{n}^{\prime}-y_{n}}<\tau_{V_{n}^{\prime}-y_{n}}\right)=P_{0}(\rho<-r) \geq c \sqrt{3} n^{-1 / 2} .
$$

Thus we have

$$
P_{y_{n}}\left(\tau_{\mathcal{S}_{1, n}}<\tau_{U_{n}}\right)=P_{y_{n}}\left(\tau_{\mathcal{S}_{1, n}}<\tau_{V_{n}^{\prime}}\right) \geq c \sqrt{3} n^{-1 / 2} .
$$

And similarly, if we look back at the harmonic measure on $\mathbb{Z}^{2}$ and let $r^{\prime}=$ $\left(C_{2}+1\right) r$. Then since

$$
\tau_{C_{2} \cdot r}<\tau_{\left\{\left(-\infty, r^{\prime}\right) \times 0\right\}}
$$

Lemma 4.2 gives us

$$
P_{0}\left(\tau_{C_{2} \cdot r}<\tau_{C}\right) \geq P_{0}\left(T_{\rho}<-r^{\prime}\right) \geq c r^{-1 / 2} .
$$

Then by Lemma 3.4,

$$
\mu_{C}(0) \geq c_{3} P_{0}\left(\tau_{C_{2} \cdot r}<\tau_{C}\right) \geq c r^{-1 / 2} .
$$

Thus we have also proved

Corollary 1. Among all connected subset $B \subset \mathbb{Z}^{2}$ containing the origin, with $r(B)=r$ and all $y \in B, \mu_{B}(y)$ is maximized (up to multiplying a constant) by when $B=[-r, r] \times 0$ and $y=(r, 0)$.

Remark 4. Note that Corollary 1, complements Kesten's paper [7], by showing that the straight line is a maximizer up to a multiplicative constant in $\mathbb{Z}^{2}$.

4.2. Spatial distribution at the escaping time. Now with Lemma 4.2 shows that a 2-dimensional simple random walk starting at $y_{n}$ will escape $B_{1}\left(y_{n},[n / 3]\right)$ before returning to $V_{n}^{\prime}$ and thus $U_{n}$ with probability at least some constant times $n^{-1 / 2}$. We next show that, given the random walk successfully escapes, it is more likely to escape from the upper half of $\mathcal{S}_{1, n}$ that the lower half of it. To make it precise, define

$$
\mathcal{S}_{1, n}^{U}=\mathcal{S}_{1, n} \cap\{(x, y), y \geq n\},
$$

and

$$
\mathcal{S}_{1, n}^{L}=\mathcal{S}_{1, n} \cap\{(x, y), y \leq n\} .
$$

Then for stopping time $\sigma=\tau_{\mathcal{S}_{1, n}} \wedge \tau_{V_{n}^{\prime}}$, we want to show

$$
P_{y_{n}}\left(\tau_{\mathcal{S}_{1, n}}<\tau_{V_{n}^{\prime}}, S_{\sigma} \in \mathcal{S}_{1, n}^{U}\right) \geq P_{y_{n}}\left(\tau_{\mathcal{S}_{1, n}}<\tau_{V_{n}^{\prime}}, S_{\sigma} \in \mathcal{S}_{1, n}^{L}\right)
$$

To show this we can again use translation invariance to move everything centered at 0 . For integer $m \geq 1$, let

$A_{m}^{+}=\left\{(x, y) \in \mathbb{Z}^{2}, x+y=m, x \in[0, m]\right\} \cup\left\{(x, y) \in \mathbb{Z}^{2},-x+y=m, x \in[-m, 0]\right\}$ 
and

$A_{m}^{-}=\left\{(x, y) \in \mathbb{Z}^{2}, x+y=-m, x \in[-m, 0],\right\} \cup\left\{(x, y) \in \mathbb{Z}^{2},-x+y=-m, x \in[0, m]\right\}$ be the upper and lower half of $\partial B_{1}(0, m)$. Then define $C_{m}^{-}=\{(0,-i), i=$ $0,1, \cdots, m\}$, and $C_{m}^{+}=\{(0, i), i=0,1, \cdots, m\}$. To show (68), it suffices to prove the following lemma:

Lemma 4.3. For all integer $m$, define set

$$
E_{m}^{-}=A_{m}^{+} \cup A_{m}^{-} \cup C_{m}^{-}
$$

and stopping time

We have

$$
\sigma_{m}^{-}=\tau_{E_{m}^{-}}=\tau_{A_{m}^{+}} \wedge \tau_{A_{m}^{-}} \wedge \tau_{C_{m}^{-}}
$$

$$
P_{0}\left(\tau_{A_{m}^{+}}=\sigma_{m}^{-}\right) \geq P_{0}\left(\tau_{A_{m}^{-}}=\sigma_{m}^{-}\right) .
$$

Proof. For

and stopping time

$$
E_{m}=A_{m}^{+} \cup A_{m}^{-} \cup C_{m}^{-} \cup C_{m}^{+},
$$

by symmetry we have

$$
\sigma_{m}=\tau_{E_{m}}=\tau_{A_{m}^{+}} \wedge \tau_{A_{m}^{-}} \wedge \tau_{C_{m}^{-}} \wedge \tau_{C_{m}^{+}},
$$

$$
P_{0}\left(\tau_{A_{m}^{+}}=\sigma_{m}\right)=P_{0}\left(\tau_{A_{m}^{-}}=\sigma_{m}\right) .
$$

At the same time,

$$
\begin{aligned}
P_{0}\left(\tau_{A_{m}^{+}}=\sigma_{m}^{-}\right) & =P_{0}\left(\tau_{A_{m}^{+}} \leq \tau_{C_{m}^{+}}, \tau_{A_{m}^{+}}=\sigma_{m}^{-}\right)+P_{0}\left(\tau_{A_{m}^{+}}>\tau_{C_{m}^{+}}, \tau_{A_{m}^{+}}=\sigma_{m}^{-}\right) \\
& =P_{0}\left(\tau_{A_{m}^{+}}=\sigma_{m}\right)+P_{0}\left(\tau_{C_{m}^{+}}<\sigma_{m}^{-}, \tau_{A_{m}^{+}}=\sigma_{m}^{-}\right),
\end{aligned}
$$

and

$$
\begin{aligned}
P_{0}\left(\tau_{A_{m}^{-}}=\sigma_{m}^{-}\right) & =P_{0}\left(\tau_{A_{m}^{-}} \leq \tau_{C_{m}^{+}}, \tau_{A_{m}^{-}}=\sigma_{m}^{-}\right)+P_{0}\left(\tau_{A_{m}^{-}}>\tau_{C_{m}^{+}}, \tau_{A_{m}^{-}}=\sigma_{m}^{-}\right) \\
& =P_{0}\left(\tau_{A_{m}^{-}}=\sigma_{m}\right)+P_{0}\left(\tau_{C_{m}^{+}}<\sigma_{m}^{-}, \tau_{A_{m}^{-}}=\sigma_{m}^{-}\right) .
\end{aligned}
$$

Thus it is sufficient to show

$$
P_{0}\left(\tau_{C_{m}^{+}}<\sigma_{m}^{-}, \tau_{A_{m}^{+}}=\sigma_{m}^{-}\right) \geq P_{0}\left(\tau_{C_{m}^{+}}<\sigma_{m}^{-}, \tau_{A_{m}^{-}}=\sigma_{m}^{-}\right) .
$$

Under event $\left\{\tau_{C_{m}^{+}}<\sigma_{m}^{-}\right\}$, let random variable $N_{m}^{+}$be the last time $S$ visits $C_{m}^{+}$in $\left[0, \sigma_{m}^{-}-1\right]$. Note that $N_{m}^{+}$is not a stopping time so we cannot use strong Markov property. But we can nonetheless have the decomposition:

$$
\begin{aligned}
& P_{0}\left(\tau_{C_{m}^{+}}<\sigma_{m}^{-}, \tau_{A_{m}^{+}}=\sigma_{m}^{-}\right) \\
& =\sum_{\substack { k=1 \\
\begin{subarray}{c}{x_{1}, x_{2}, \cdots, x_{k-1} \notin E_{m}^{-} \\
x_{k} \in x_{k} \in C_{m}^{+} \backslash\{0,(0, m)\}{ k = 1 \\
\begin{subarray} { c } { x _ { 1 } , x _ { 2 } , \cdots , x _ { k - 1 } \notin E _ { m } ^ { - } \\
x _ { k } \in x _ { k } \in C _ { m } ^ { + } \backslash \{ 0 , ( 0 , m ) \} } }\end{subarray}}^{\sum_{0}^{\infty}} P_{0}\left(S_{1}=x_{1}, \cdots, S_{k}=x_{k}, N_{m}^{+}=k, \tau_{C_{m}^{+}}<\sigma_{m}^{-}, \tau_{A_{m}^{+}}=\sigma_{m}^{-}\right) .
\end{aligned}
$$


and

(74)

$$
\begin{aligned}
& P_{0}\left(\tau_{C_{m}^{+}}<\sigma_{m}^{-}, \tau_{A_{m}^{-}}=\sigma_{m}^{-}\right) \\
& =\sum_{k=1}^{\infty} \sum_{x_{1}, x_{2}, \cdots, x_{k-1} \notin E_{m}^{-}} P_{0}\left(S_{1}=x_{1}, \cdots, S_{k}=x_{k}, N_{m}^{+}=k, \tau_{C_{m}^{+}}<\sigma_{m}^{-}, \tau_{A_{m}^{-}}=\sigma_{m}^{-}\right) . \\
& x_{k} \in x_{k} \in C_{m}^{+} \backslash\{0,(0, m)\}
\end{aligned}
$$

Note that for each $k, x_{1}, x_{2}, \cdots, x_{k-1} \notin E_{m}^{-}$, and $x_{k} \in x_{k} \in C_{m}^{+} \backslash\{0,(0, m)\}$, we have

$$
\left\{S_{1}=x_{1}, \cdots, S_{k}=x_{k}, N_{m}^{+}=k, \tau_{C_{m}^{+}}<\sigma_{m}^{-}, \tau_{A_{m}^{+}}=\sigma_{m}^{-}\right\}
$$

$=\left\{S_{1}=x_{1}, \cdots, S_{k}=x_{k}, S_{k+1+}\right.$. visit $A_{m}^{+}$no later than it first visits $\left.A_{m}^{-} \cup C_{m}^{+} \cup C_{m}^{-}\right\}$.

So by Markov property, we have

$$
\begin{aligned}
& P_{0}\left(S_{1}=x_{1}, \cdots, S_{k}=x_{k}, N_{m}^{+}=k, \tau_{C_{m}^{+}}<\sigma_{m}^{-}, \tau_{A_{m}^{+}}=\sigma_{m}^{-}\right) \\
& =P_{0}\left(S_{1}=x_{1}, \cdots, S_{k}=x_{k}\right) P_{x_{k}}\left(\tau_{A_{m}^{+}}=\sigma_{m}\right) .
\end{aligned}
$$

Plugging back in (73) we have

$$
\begin{aligned}
& P_{0}\left(\tau_{C_{m}^{+}}<\sigma_{m}^{-}, \tau_{A_{m}^{+}}=\sigma_{m}^{-}\right) \\
& =\sum_{k=1}^{\infty} \sum_{\substack{x_{1}, x_{2}, \cdots, x_{k-1} \notin E_{m}^{-} \\
x_{k} \in x_{k} \in C_{m}^{+} \backslash\{0,(0, m)\}}} P_{0}\left(S_{1}=x_{1}, \cdots, S_{k}=x_{k}\right) P_{x_{k}}\left(\tau_{A_{m}^{+}}=\sigma_{m}\right),
\end{aligned}
$$

while the same argument for $A_{m}^{-}$gives us

$$
\begin{aligned}
& P_{0}\left(\tau_{C_{m}^{+}}<\sigma_{m}^{-}, \tau_{A_{m}^{-}}=\sigma_{m}^{-}\right) \\
& =\sum_{k=1}^{\infty} \sum_{\substack{x_{1}, x_{2}, \cdots, x_{k-1} \notin E_{m}^{-} \\
x_{k} \in x_{k} \in C_{m}^{+} \backslash\{0,(0, m)\}}} P_{0}\left(S_{1}=x_{1}, \cdots, S_{k}=x_{k}\right) P_{x_{k}}\left(\tau_{A_{m}^{-}}=\sigma_{m}\right) .
\end{aligned}
$$

Comparing (76) and (77) term by term, one can see it suffices to show that for all $z=(0, j) \in x_{k} \in C_{m}^{+} \backslash\{0,(0, m)\}$,

$$
P_{z}\left(\tau_{A_{m}^{+}}=\sigma_{m}\right) \geq P_{z}\left(\tau_{A_{m}^{-}}=\sigma_{m}\right) .
$$

To show (78), one first sees that under $\left\{\tau_{A_{m}^{+}}=\sigma_{m}\right\}$ or $\left\{\tau_{A_{m}^{-}}=\sigma_{m}\right\}$, a random walk starting at $z$ has to move horizontally at the first step then remain in the right or left half triangle of $B_{1}(0, m)$ until it exits from $A_{m}^{+}$or $A_{m}^{-}$. Then for all integer $i \in[0, m]$ we define

$$
\begin{gathered}
C_{m, i}=\{(0, y), 2 i-m \leq y \leq m\} \\
A_{m, i}^{+, r}=\left\{(x, y) \in \mathbb{Z}^{2}, x+y=m, x \in[0, m-i]\right\}
\end{gathered}
$$


and

$$
A_{m, i}^{-, r}=\cup\left\{(x, y) \in \mathbb{Z}^{2},-x+y=2 i-m, x \in[0, m-i]\right\} .
$$

Now we have by symmetry

$$
P_{z}\left(\tau_{A_{m}^{+}}=\sigma_{m}\right)=\frac{1}{2} P_{(1, j)}\left(\bar{\tau}_{A_{m, 0}^{+, r}} \leq \bar{\tau}_{A_{m, 0}^{-, r}}, \bar{\tau}_{A_{m, 0}^{+, r}} \leq \bar{\tau}_{C_{m, 0}}\right) .
$$

and

$$
P_{z}\left(\tau_{A_{m}^{-}}=\sigma_{m}\right)=\frac{1}{2} P_{(1, j)}\left(\bar{\tau}_{A_{m, 0}^{-, r}} \leq \bar{\tau}_{A_{m, 0}^{+, r}}, \bar{\tau}_{A_{m, 0}^{-, r}} \leq \bar{\tau}_{C_{m, 0}}\right) .
$$

The right hand side of the Equation (80) equals to 0 when $j=m-1$. Otherwise, note that if a random walk starting from $(1, j)$ want to visit $A_{m, 0}^{-, r}$ before visiting $A_{m, 0}^{+, r}$ or $C_{m, 0}$, it has to first get through $A_{m, j}^{-, r}$ before visiting $A_{m, 0}^{+, r}$ or $C_{m, 0}$. Thus

$$
P_{(1, j)}\left(\bar{\tau}_{A_{m, 0}^{-, r}} \leq \bar{\tau}_{A_{m, 0}^{+, r}}, \bar{\tau}_{A_{m, 0}^{-, r}} \leq \bar{\tau}_{C_{m, 0}}\right) \leq P_{(1, j)}\left(\bar{\tau}_{A_{m, j}^{-, r}} \leq \bar{\tau}_{A_{m, 0}^{+, r}}, \bar{\tau}_{A_{m, j}^{-, r}} \leq \bar{\tau}_{C_{m, 0}}\right) .
$$

Then note that in order to have a random walk starting from $(1, j)$ get to $A_{m, j}^{-, r}$ before visiting $A_{m, 0}^{+, r}$ or $C_{m, 0}$, it only need to avoid $A_{m, j}^{+, r}$ and $C_{m, j}$. So we have

$$
P_{(1, j)}\left(\bar{\tau}_{A_{m, j}^{-, r}} \leq \bar{\tau}_{A_{m, 0}^{+, r}}, \bar{\tau}_{A_{m, j}^{-, r}} \leq \bar{\tau}_{C_{m, 0}}\right)=P_{(1, j)}\left(\bar{\tau}_{A_{m, j}^{-, r}} \leq \bar{\tau}_{A_{m, j}^{+, r}}, \bar{\tau}_{A_{m, j}^{-, r}} \leq \bar{\tau}_{C_{m, j}}\right) .
$$

By symmetry one can see

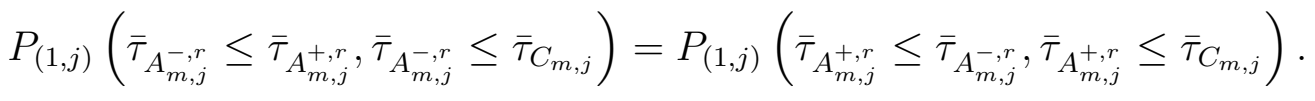

Moreover, note that a random walk starting from $(1, j)$ must exist the smaller triangle bounded by $A_{m, j}^{+, r}, A_{m, j}^{-, r}$, and $C_{m, j}$ before exiting the larger on bounded by $A_{m, 0}^{+, r}, A_{m, 0}^{-, r}$, and $C_{m, 0}$. I.e.,

$$
\sigma_{j}^{r}=\bar{\tau}_{A_{m, j}^{+, r}} \wedge \bar{\tau}_{A_{m, j}^{-, r}} \wedge \tau_{C_{m, j}} \leq \sigma^{r}=\bar{\tau}_{A_{m, 0}^{+, r}} \wedge \bar{\tau}_{A_{m, 0}^{-, r}} \wedge \tau_{C_{m, 0}}
$$

Thus

$$
\begin{aligned}
P_{(1, j)} & \left(\bar{\tau}_{A_{m, j}^{+, r}} \leq \bar{\tau}_{A_{m, j}^{-, r}}, \bar{\tau}_{A_{m, j}^{+, r}} \leq \bar{\tau}_{C_{m, j}}\right) \\
& =P_{(1, j)}\left(\bar{\tau}_{A_{m, j}^{+, r}} \leq \sigma_{j}^{r}\right) \\
& \leq P_{(1, j)}\left(\bar{\tau}_{A_{m, j}^{+, r}} \leq \sigma^{r}\right) \\
& \leq P_{(1, j)}\left(\bar{\tau}_{A_{m, j}^{+, r}} \leq \bar{\tau}_{A_{m, 0}^{-, r}} \wedge \tau_{C_{m, 0}}\right) \\
& =P_{(1, j)}\left(\bar{\tau}_{A_{m, j}^{+, r}} \leq \bar{\tau}_{A_{m, 0}^{-, r}}, \bar{\tau}_{A_{m, j}^{+, r}} \leq \bar{\tau}_{C_{m, 0}}\right) \\
& \leq P_{(1, j)}\left(\bar{\tau}_{A_{m, 0}^{+, r}} \leq \bar{\tau}_{A_{m, 0}^{-, r}}, \bar{\tau}_{A_{m, j}^{+, r}} \leq \bar{\tau}_{C_{m, 0}}\right) .
\end{aligned}
$$

Finally note that the right hand side of the last inequality in (81) is exactly the right hand side of (79). 
With Lemma 4.3, we immediately get (68) from translation invariance.

4.3. Proof of Theorem 2. Now we have all the tools we need to finish the proof of Theorem 2. Recall (49) and apply it to $U_{n}$ and $y_{n}$,

$H_{U_{n}, N}\left(y_{n}\right)$

$=E_{y_{n}}\left[\right.$ number of visits to $L_{N}$ in time interval $\left.\left[0, \tau_{U_{n}}\right]\right]$

$=\sum_{w \in L_{N}} P_{y_{n}}\left(\tau_{L_{N}}<\tau_{U_{n}}, S_{\tau_{L_{N}}}=w\right) E_{w}\left[\right.$ number of visits to $L_{N}$ in time interval $\left.\left[0, \tau_{U_{n}}\right]\right]$.

Note that for all $w \in L_{N}$,

$$
P_{w}\left(\tau_{L_{n}} \leq \tau_{U_{n}}\right)=1
$$

We have

$$
\begin{aligned}
& H_{U_{n}, N}\left(y_{n}\right) \\
& \geq \sum_{w \in L_{N}} P_{y_{n}}\left(\tau_{L_{N}}<\tau_{U_{n}}, S_{\tau_{L_{N}}}=w\right) E_{w}\left[\text { number of visits to } L_{N} \text { in time interval }\left[0, \tau_{L_{n}}\right]\right] \\
& =4 P_{y_{n}}\left(\tau_{L_{N}}<\tau_{U_{n}}\right)(N-n) .
\end{aligned}
$$

Then according again to strong Markov property and the fact that a random walk starting from $y_{n}$ has to visit $L_{2 n}$ before $L_{N}$,

$$
P_{y_{n}}\left(\tau_{L_{N}}<\tau_{U_{n}}\right)=\sum_{w \in L_{2 n}} P_{y_{n}}\left(\tau_{L_{2 n}}<\tau_{U_{n}}, S_{\tau_{L_{2 n}}}=w\right) P_{w}\left(\tau_{L_{N}}<\tau_{U_{n}}\right) .
$$

Again, note that for all $w \in L_{2 n}$

$$
P_{w}\left(\tau_{L_{N}}<\tau_{U_{n}}\right) \geq P_{w}\left(\tau_{L_{N}}<\tau_{L_{n}}\right)=\frac{n}{N-n} .
$$

Thus to prove Theorem 2 it is sufficient to show that for $N$ sufficiently larger than $n$,

$$
P_{y_{n}}\left(\tau_{L_{2 n}}<\tau_{U_{n}}\right) \geq c n^{-1 / 2}
$$

To show (82), we have

$$
\begin{aligned}
P_{y_{n}}\left(\tau_{L_{2 n}}<\tau_{U_{n}}\right) & \geq \sum_{w \in \mathcal{S}_{1, n}^{U}} P_{y_{n}}\left(\tau_{\mathcal{S}_{1, n}}<\tau_{V_{n}}, S_{\tau_{\mathcal{S}_{1, n}}}=w \in \mathcal{S}_{1, n}^{U}\right) P_{w}\left(\tau_{L_{2 n}}<\tau_{U_{n}}\right) \\
& =\sum_{w \in \mathcal{S}_{1, n}^{U}} P_{y_{n}}\left(\tau_{\mathcal{S}_{1, n}}<\tau_{V_{n}^{\prime}}, S_{\sigma}=w \in \mathcal{S}_{1, n}^{U}\right) P_{w}\left(\tau_{L_{2 n}}<\tau_{U_{n}}\right) .
\end{aligned}
$$

Note that by invariance principle there is a constant $c$ such that for any sufficiently large $n$ and $w \in \mathcal{S}_{1, n}^{U}$,

$$
P_{w}\left(\tau_{L_{2 n}}<\tau_{U_{n}}\right) \geq c .
$$

Thus

$$
P_{y_{n}}\left(\tau_{L_{2 n}}<\tau_{U_{n}}\right) \geq c P_{y_{n}}\left(\tau_{\mathcal{S}_{1, n}}<\tau_{V_{n}^{\prime}}, S_{\sigma}=w \in \mathcal{S}_{1, n}^{U}\right) .
$$


Then by (67) and (68), we have

$$
P_{y_{n}}\left(\tau_{\mathcal{S}_{1, n}}<\tau_{V_{n}^{\prime}}, S_{\sigma} \in \mathcal{S}_{1, n}^{U}\right) \geq \frac{1}{2} P_{y_{n}}\left(\tau_{\mathcal{S}_{1, n}}<\tau_{V_{n}^{\prime}}\right) \geq c n^{-1 / 2} .
$$

Thus, the proof of Theorem 2 is complete.

\section{TOtAl HARMONiC MEASURE ON FINITE SETS}

5.1. Upper bound in Theorem 3. To show the upper bound in (9), without loss of generality we can assume $B \cap L_{0} \neq \varnothing$, which implies that $\min _{x \in B}\left\{x_{2}\right\}=0$. Otherwise, for $x_{0}=\left(x_{1,0}, x_{2,0}\right)$ that has the smallest height in $B$, define

$$
B^{\prime}=B \cup\left\{\left(x_{1,0}, j\right), j=0,1, \cdots, x_{2,0}-1\right\} .
$$

By Proposition 2, we have $H_{B^{\prime}} \geq H_{B}$ and $\left|B^{\prime}\right|=|B|+\min _{x \in B}\left\{x_{2}\right\}$. Thus it suffices for us to prove that for any connected and finite $B$ with $B \cap L_{0} \neq \varnothing$,

$$
H_{B} \leq C|B| \text {. }
$$

And we prove (85) inductively. When $|B|=1$, we have proved the desired upper bound in (17). Suppose we have proved (85) for all connected $B$ with $|B| \leq n$, $B \cap L_{0} \neq \varnothing$. Then for a $B$ such that $|B|=n+1, B \cap L_{0} \neq \varnothing$, we first show that one can remove one vertex in $B$ and still have a connected subset intersecting $L_{0}$. In fact we prove something even stronger:

Lemma 5.1. For any finite and connected $B \subset \mathbb{Z}^{2}$ with $|B| \geq 2$, there are always two points $x_{1}, x_{2} \in B$ such that $B \backslash\left\{x_{1}\right\}$ and $B \backslash\left\{x_{2}\right\}$ are both connected.

Remark 5. With Lemma 5.1, we can make sure that starting from $|B|=n+1$, $B \cap L_{0} \neq \varnothing$, we can remove one point and it will not be in $L_{0}$ if $\left|B \cap L_{0}\right|=1$. Thus the new connected subset still intersects $L_{0}$.

Proof. Again, we prove this lemma by induction. For $|B|=2$ or $|B|=3$, it is easy to check the lemma holds. Now suppose it also holds for all connected $|B| \leq n$. Then from the assumption we also have that

Observation 1: for any connected $B$ such that $|B| \leq n$ and any $x_{0} \in B^{c}$ such that $d\left(x_{0}, B\right)=1$, where

$$
d(x, B)=\inf _{y \in B}\{|x-y|\}
$$

there must exists an $x \in B$ such that $B \backslash\{x\}$ is connected while $d\left(x_{0}, B \backslash\{x\}\right)=1$.

To see this, note that if

$$
\left|\left\{y \in B:\left|x_{0}-y\right|=1\right\}\right| \geq 2
$$

then removing one point will not change the distance between $x$ and the smaller subset. So either $x_{1}$ or $x_{2}$ in the inductive assumption is good. Otherwise, let $y_{0}$ be the only point in $B$ neighboring $x_{0}$. By the inductive assumption we have two points $x_{1}$ and $x_{2}$ which we can remove, and one of them must not be $y_{0}$. Thus we still have an $x \in B$ such that $B \backslash\{x\}$ is connected while $d\left(x_{0}, B \backslash\{x\}\right)=1$. 
With the observation above, now for any connected $B$ such that $|B|=n+1$, we first choose one point $y$ arbitrarily from $B$. If $B \backslash\{y\}$ is connected, note that $|B \backslash\{y\}|=n$ and that $d(y, B \backslash\{y\})=1$. Our observation above shows that there must be a $y^{\prime} \in B \backslash\{y\}$ such that $B \backslash\left\{y, y^{\prime}\right\}$ is also connected and

$$
d\left(y, B \backslash\left\{y, y^{\prime}\right\}\right)=1 \text {. }
$$

This implies that $B \backslash\left\{y^{\prime}\right\}=B \backslash\left\{y, y^{\prime}\right\} \cup\{y\}$ is connected. And we have found our two "removable" points. Otherwise, if $B \backslash\{y\}$ is not connected, it must have at least two connected components, say $B_{1}$ and $B_{2}$.

Remark 6. If $B \backslash\{y\}$ has more than two connected components, just choose two of them arbitrarily.

Let

$$
d(A, B)=\inf _{x \in A, y \in B}\{|x-y|\}
$$

for all finite $A$ and $B$. Noting that $B$ is connected, we must have $d\left(B_{1}, B \backslash B_{1}\right)=1$. But since $B_{1}$ is not connected to $B \backslash\left(B_{1} \cup\{y\}\right)$, we also have

$$
d\left(B_{1}, B \backslash\left(B_{1} \cup\{y\}\right)\right) \geq 2 .
$$

Thus one can now see $d\left(y, B_{1}\right)=1$ and $d\left(y, B_{2}\right)=1$. Then note that $\left|B_{1}\right|$ and $\left|B_{2}\right|$ are both less than $n$. So by Observation 1 we again have there is a $x_{1} \in B_{1}$ such that $B_{1} \backslash\left\{x_{1}\right\}$ is connected and that

$$
d\left(y, B_{1} \backslash\left\{x_{1}\right\}\right)=1,
$$

which implies that $\left(B_{1} \backslash\left\{x_{1}\right\}\right) \cup\{y\}$ is connected,

$$
d\left(\left(B_{1} \backslash\left\{x_{1}\right\}\right) \cup\{y\}, B_{2}\right)=1,
$$

and that $B \backslash\left\{x_{1}\right\}=\left(B_{1} \backslash\left\{x_{1}\right\}\right) \cup\{y\} \cup B_{2}$ is connected. The same argument on $B_{2}$ also gives that there is a $x_{2} \in B_{2}$ such that $B \backslash\left\{x_{2}\right\}$ is connected. Finally note that $B_{1}$ and $B_{2}$ are different connected component, which implies that $B_{1} \cap B_{2}=\varnothing$. So we have $x_{1} \neq x_{2}$ and the proof of this lemma is complete.

With Lemma 5.1, we continue with the inductive argument for the growth rate of $H_{B}$. For any finite and connected $B$ such that $|B|=n+1, B \cap L_{0} \neq \varnothing$, there has to be an $x=\left(x_{1}, x_{2}\right) \in B$ such that $\tilde{B}=B \backslash\{x\}$ is still connected and $\tilde{B} \cap L_{0} \neq \varnothing$. By inductive assumption we know that $H_{\tilde{B}} \leq C n$. So now we can concentrate on comparing $H_{\tilde{B}}$ and $H_{B}$.

Since $B$ is finite, for any $v \in B$ sufficiently large $N$ we have

$$
H_{B, N}(v)=\sum_{z \in L_{N}} P_{z}\left(\tau_{v}=\tau_{B \cup L_{0}}\right) .
$$

And thus

$$
H_{B, N}=\sum_{v \in B} H_{B, N}(v)=\sum_{z \in L_{N}} P_{z}\left(\tau_{B} \leq \tau_{L_{0}}\right)
$$


while

$$
H_{\tilde{B}, N}=\sum_{z \in L_{N}} P_{z}\left(\tau_{\tilde{B}} \leq \tau_{L_{0}}\right)
$$

Note that for each $z \in L_{N}$,

$$
P_{z}\left(\tau_{B} \leq \tau_{L_{0}}\right)-P_{z}\left(\tau_{\tilde{B}} \leq \tau_{L_{0}}\right)=P_{z}\left(\tau_{x} \leq \tau_{L_{0}}<\tau_{\tilde{B}}\right) .
$$

Moreover, by strong Markov property,

$$
P_{z}\left(\tau_{x} \leq \tau_{L_{0}}<\tau_{\tilde{B}}\right)=P_{z}\left(\tau_{x}=\tau_{B \cup L_{0}}\right) P_{x}\left(\tau_{L_{0}}<\tau_{\tilde{B}}\right) .
$$

Combining (86) and (87), we have

$$
\begin{aligned}
H_{B, N}-H_{\tilde{B}, N} & =P_{x}\left(\tau_{L_{0}}<\tau_{\tilde{B}}\right) \sum_{z \in L_{N}} P_{z}\left(\tau_{x}=\tau_{B \cup L_{0}}\right) \\
& =P_{x}\left(\tau_{L_{0}}<\tau_{\tilde{B}}\right) H_{B, N}(x) .
\end{aligned}
$$

If $x_{2}=0$, note that in (18) we have $H_{B, N}(x) \leq 1$, which implies that $H_{B, N}-H_{\tilde{B}, N} \leq$ 1. And for $x_{2} \geq 1$, we have by Theorem 1

$$
H_{B, N}-H_{\tilde{B}, N} \leq C \sqrt{x_{2}} P_{x}\left(\tau_{L_{0}}<\tau_{\tilde{B}}\right) .
$$

And since $\tilde{B}$ is connected. There must be a finite nearest neighbor path

$$
\tilde{\mathcal{P}}_{x}=\left\{x=\tilde{P}_{0}, \tilde{P}_{1}, \tilde{P}_{2}, \cdots, \tilde{P}_{k_{n}} \in L_{0}\right\}
$$

connecting $x$ and $L_{0}$, where $\left|\tilde{P}_{i}-\tilde{P}_{i+1}\right|=1$. And since $d\left(x, L_{0}\right)=x_{2},\left|x-P_{k_{n}}\right| \geq x_{2}$. Define

$$
\tilde{m}_{x}=\inf \left\{i:\left|\tilde{P}_{i}-x\right| \geq x_{2}\right\}
$$

and

$$
\tilde{\mathcal{Q}}_{x}=\left\{\tilde{P}_{1}, \tilde{P}_{2}, \cdots, \tilde{P}_{\tilde{m}_{x}}\right\} .
$$

One can immediately see that $\tilde{\mathcal{Q}}_{x} \subset \tilde{B}$ and that

$$
\tilde{\mathcal{Q}}_{x} \subset B\left(x, 2 x_{2}\right) .
$$

Thus

$$
\frac{1}{4} P_{x}\left(\tau_{L_{0}}<\tau_{\tilde{B}}\right) \leq \frac{1}{4} P_{x}\left(\tau_{L_{0}}<\tau_{\tilde{\mathcal{Q}}_{x}}\right) \leq P_{\tilde{P}_{1}}\left(\tau_{L_{0}}<\tau_{\tilde{\mathcal{Q}}_{x}}\right) .
$$

Then for sufficiently large $x_{2}$, recalling the $C_{2}$ defined in Lemma 3.3, let $r=x_{2} / 2 C_{2}$ and $D_{x}=\tilde{\mathcal{Q}}_{x} \cap B\left(\tilde{P}_{1}, r\right)$. Note that for sufficiently large $x_{2}$, a random walk starting at $\tilde{P}_{1}$ has to hit $\partial^{\text {out }} B\left(\tilde{P}_{1}, C_{2} \cdot r\right)$ before reaching $L_{0}$. We have by (1), (2) and Lemma 3.4 ,

$$
\begin{aligned}
P_{\tilde{P}_{1}}\left(\tau_{L_{0}}<\tau_{\tilde{\mathcal{Q}}_{x}}\right) & \leq P_{\tilde{P}_{1}}\left(\tau_{\partial^{\text {out }} B\left(x, C_{2} \cdot r\right)}<\tau_{\tilde{\mathcal{Q}}_{x} \cap B\left(x, C_{2} \cdot r\right)}\right) \\
& \leq P_{\tilde{P}_{1}}\left(\tau_{\partial^{\text {out }} B\left(x, C_{2} \cdot r\right)}<\tau_{D_{x}}\right) \\
& \leq c_{3}^{-1} \mu_{D_{x}-\tilde{P}_{1}}(0) \leq C x_{2}^{-1 / 2} \\
& 28
\end{aligned}
$$


Combining (89) and (90) we have that there is a constant $C$ independent to $n, N$ and $x$, such that

$$
H_{B, N}-H_{\tilde{B}, N} \leq C .
$$

Thus the proof of (9) is complete.

5.2. Lower bound in Theorem 3. First, (11) is obvious. Now we show the lower bound in (10). Since $B$ is finite, let $\bar{x}=\left(\bar{x}_{1}, \bar{x}_{2}\right)$ be a point in $B$ such that

$$
\bar{x}_{2}=\max _{x \in B}\left\{x_{2}\right\} .
$$

Note that by Proposition $2, H_{B} \geq H_{\bar{x}}$. It suffices to prove (10) for the single element set $\{\bar{x}\}$. Recall that

$$
\begin{aligned}
H_{\{\bar{x}\}, N} & =E_{\bar{x}}\left[\text { number of visits to } L_{N} \text { in }\left[0, \tau_{\{\bar{x}\} \cup L_{0}}\right]\right] \\
& \geq P_{\bar{x}}\left(\tau_{L_{N}}<\tau_{\{\bar{x}\} \cup L_{0}}\right) \inf _{z \in L_{N}} E_{z}\left[\text { number of visits to } L_{N} \text { in }\left[0, \tau_{\{\bar{x}\} \cup L_{0}}\right]\right]
\end{aligned}
$$

and that for sufficiently large $N$ and any $z \in L_{N}$,

$$
\begin{aligned}
& E_{z}\left[\text { number of visits to } L_{N} \text { in }\left[0, \tau_{\{\bar{x}\} \cup L_{0}}\right]\right] \\
\geq & E_{z}\left[\text { number of visits to } L_{N} \text { in }\left[0, \tau_{L_{x_{2}}}\right]\right]=4\left(N-x_{2}\right) \geq 2 N .
\end{aligned}
$$

To prove (10) it is sufficient to show that for sufficiently large $x_{2}$

$$
P_{\bar{x}}\left(\tau_{L_{N}}<\tau_{\{\bar{x}\} \cup L_{0}}\right) \geq \frac{c \bar{x}_{2}}{N \log \left(\bar{x}_{2}\right)} .
$$

Now let $n_{\bar{x}}$ be the largest odd number less than $\bar{x}_{2}$. We define $B_{1}\left(\bar{x}, n_{\bar{x}}\right)$ be the $L_{1}$ ball centered at $x$ with radius $n_{x}$. Moreover we define

$$
W_{\bar{x}}^{1,+}=\partial B_{1}\left(\bar{x}, n_{\bar{x}}\right) \cap\left\{\left(y_{1}, y_{2}\right), y_{2} \geq \bar{x}_{2}+\left(n_{\bar{x}}+1\right) / 2\right\}
$$

be the upper corner of $\partial B_{1}\left(\bar{x}, n_{\bar{x}}\right)$. By symmetry we have

$$
P_{\bar{x}}\left(\tau_{\partial B_{1}\left(\bar{x}, n_{\bar{x}}\right)}<\tau_{\bar{x}}, S_{\tau_{\partial B_{1}\left(\bar{x}, n_{\bar{x}}\right)}} \in W_{\bar{x}}^{1,+}\right)=\frac{1}{4} P_{\bar{x}}\left(\tau_{\partial B_{1}\left(\bar{x}, n_{\bar{x}}\right)}<\tau_{\bar{x}}\right) .
$$

Thus we have

$$
\begin{aligned}
& P_{\bar{x}}\left(\tau_{L_{N}}<\tau_{\{\bar{x}\} \cup L_{0}}\right) \\
& \quad \geq P_{\bar{x}}\left(\tau_{\partial B_{1}\left(\bar{x}, n_{\bar{x}}\right)}<\tau_{\bar{x}}, S_{\tau_{\partial B_{1}\left(\bar{x}, n_{\bar{x}}\right)}} \in W_{\bar{x}}^{1,+}\right) \inf _{y \in W_{\bar{x}}^{1,+}} P_{y}\left(\tau_{L_{N}}<\tau_{L_{\bar{x}_{2}}}\right) \\
& \quad \geq \frac{1}{4} P_{\bar{x}}\left(\tau_{\partial B_{1}\left(\bar{x}, n_{\bar{x}}\right)}<\tau_{\bar{x}}\right) \inf _{y \in W_{\bar{x}}^{1,+}} P_{y}\left(\tau_{L_{N}}<\tau_{L_{\bar{x}_{2}}}\right) .
\end{aligned}
$$

Then note that for any $y \in W_{\bar{x}}^{1,+}$

$$
P_{y}\left(\tau_{L_{N}}<\tau_{L_{\bar{x}_{2}}}\right) \geq \frac{n_{\bar{x}}}{2 N} \geq \frac{\bar{x}_{2}}{4 N} .
$$

Thus it is sufficient for us to prove that 
Lemma 5.2. There is a constant $c>0$ such that for all sufficiently large $\bar{x}_{2}$,

$$
P_{\bar{x}}\left(\tau_{\partial B_{1}\left(\bar{x}, n_{\bar{x}}\right)}<\tau_{\bar{x}}\right) \geq \frac{c}{\log \left(\bar{x}_{2}\right)} .
$$

Proof. For $S_{n}=\left(S_{1, n}, S_{2, n}\right)$ to be the simple random walk starting at $\bar{x}$, consider the martingale

$$
M_{n}=\left(S_{2, n}-\bar{x}_{2}\right)^{2}-\frac{n}{2} .
$$

Note that $M_{0}=0$, so we have

$$
E_{\bar{x}}\left[\tau_{\partial B_{1}\left(\bar{x}, n_{\bar{x}}\right)}\right] \leq \sup _{y \in \partial B_{1}\left(\bar{x}, n_{\bar{x}}\right)}\left(y_{2}-\bar{x}_{2}\right)^{2} \leq \bar{x}_{2}^{2} .
$$

Thus

$$
P_{\bar{x}}\left(\tau_{\partial B_{1}\left(\bar{x}, n_{\bar{x}}\right)} \geq x_{2}^{3}\right) \leq \frac{1}{x_{2}} .
$$

On the other hand, for simple random walk in $\mathbb{Z}^{2}$ it was shown in [5] and [6] that for sufficiently large $x_{2}$,

$$
P_{\bar{x}}\left(\tau_{\bar{x}}>\bar{x}_{2}^{3}\right)=\frac{\pi}{\log \left(\bar{x}_{2}^{3}\right)}+O\left(\frac{1}{\log ^{2}\left(\bar{x}_{2}^{3}\right)}\right) \geq \frac{\pi}{6 \log \left(\bar{x}_{2}\right)} .
$$

Thus note that

$$
\begin{aligned}
P_{\bar{x}}\left(\tau_{\partial B_{1}\left(\bar{x}, n_{\bar{x}}\right)}<\tau_{\bar{x}}\right) & \geq P_{\bar{x}}\left(\tau_{\partial B_{1}\left(\bar{x}, n_{\bar{x}}\right)}<\bar{x}_{2}^{3}, \tau_{\bar{x}}>\bar{x}_{2}^{3}\right) \\
& =P_{\bar{x}}\left(\tau_{\bar{x}}>\bar{x}_{2}^{3}\right)-P_{\bar{x}}\left(\tau_{\partial B_{1}\left(\bar{x}, n_{\bar{x}}\right)} \geq \bar{x}_{2}^{3}, \tau_{\bar{x}}>\bar{x}_{2}^{3}\right) \\
& \geq P_{\bar{x}}\left(\tau_{\bar{x}}>\bar{x}_{2}^{3}\right)-P_{\bar{x}}\left(\tau_{\partial B_{1}\left(\bar{x}, n_{\bar{x}}\right)} \geq \bar{x}_{2}^{3}\right) .
\end{aligned}
$$

Combining (94) and (95), we have for sufficiently large $x_{2}$,

$$
P_{\bar{x}}\left(\tau_{\partial B_{1}\left(\bar{x}, n_{\bar{x}}\right)}<\tau_{\bar{x}}\right) \geq \frac{\pi}{6 \log \left(\bar{x}_{2}\right)}-\frac{1}{x_{2}} \geq \frac{\pi}{7 \log \left(\bar{x}_{2}\right)}
$$

which finished the proof of this lemma.

With Lemma 5.2, the proof of (10) and thus Theorem 3 is complete.

5.3. Proof of Theorem 4. Now we show that the total harmonic measure is maximized (up to multiplying a constant) by the vertical line segment $V_{n}$ over all connected finite subsets with the same cardinality and connected to $L_{0}$. And again we do this inductively. By (88), we have

$$
H_{V_{n}, N}-H_{V_{n-1}, N}=H_{V_{n}, N}\left(y_{n}\right) P_{y_{n}}\left(\tau_{L_{0}}<\tau_{V_{n-1}}\right) .
$$

According to Theorem 2, we have

$$
H_{V_{n}, N}\left(y_{n}\right) \geq x \sqrt{n} .
$$

Noting that

$$
P_{y_{n}}\left(\tau_{L_{0}}<\tau_{V_{n-1}}\right) \underset{30}{\geq} P_{y_{n}}\left(\tau_{L_{0}}<\tau_{V_{n}}\right)
$$


it suffices to prove that

$$
P_{y_{n}}\left(\tau_{L_{0}}<\tau_{V_{n}}\right) \geq \frac{c}{\sqrt{n}}
$$

On the other hand, recall that

$$
\mathcal{S}_{1, n}=\partial B_{1}\left(y_{n},[n / 3]\right)
$$

and that

$$
\mathcal{S}_{1, n}^{U}=\mathcal{S}_{1, n} \cap\{(x, y), y \geq n\} .
$$

We have

$$
P_{y_{n}}\left(\tau_{L_{0}}<\tau_{V_{n}}\right) \geq P_{y_{n}}\left(\tau_{\mathcal{S}_{1, n}^{U}}<\tau_{V_{n}}\right) \inf _{y \in \mathcal{S}_{1, n}^{U}} P_{y}\left(\tau_{L_{0}}<\tau_{V_{n}}\right) .
$$

Again by invariant principle, there is a constant $c>0$ such that for any $n$ and $y \in \mathcal{S}_{1, n}^{U}$,

And then by (67) and (68),

$$
P_{y}\left(\tau_{L_{0}}<\tau_{V_{n}}\right) \geq c
$$

$$
P_{y_{n}}\left(\tau_{\mathcal{S}_{1, n}^{U}}<\tau_{V_{n}}\right) \geq \frac{c}{\sqrt{n}}
$$

Thus the proof of Theorem 4 is complete.

\section{Construction and growth estimate of DLA in $\mathcal{H}$}

6.1. Construction of a growth model. With the upper bounds of the harmonic measure on the upper half plane, in this section we construct pure growth models which can be used as a dominating process for both the DLA model in $\mathcal{H}$ and the stationary DLA model that will be introduced in a follow up paper. Consider an interacting particle system $\bar{\xi}_{t}$ defined on $\{0,1\}^{\mathcal{H}}$ where $\mathcal{H}$ is the upper half plane with 1 standing for a site occupied while 0 for vacant, with transition rates as follows:

(i) For each occupied site $x=\left(x_{1}, x_{2}\right) \in \mathcal{H}$, if $x_{2}>0$ it will try to give birth to each of its nearest neighbors at a Poisson rate of $\sqrt{x_{2}}$. If $x_{2}=0$, it will try to give birth to each of its nearest neighbors at a Poisson rate of 1 .

(ii) When $x$ attempts to give birth to its nearest neighbors $y$ already occupied, the birth is suppressed.

We prove that an interacting particle system determined by the dynamic above is well-defined.

Proposition 3. The interacting particle system $\bar{\xi}_{t} \in\{0,1\}^{\mathcal{H}}$ satisfying (i) and (ii) is well defined.

Proof. The proof of Proposition 3 uses a similar idea as in Theorem 2.1 of [4]. Although here the transition rates are no longer translation invariant or uniformly bounded, we will be able to use more elaborate argument and show that with high probability the time moving forward at each step goes to 0 while still being 
un-summable all together. The next idea is very similar to Borel-Cantelli lemma. However, rather than using the result directly, we will have the proof of BorelCantelli lemma embedded in our argument. By doing so, we will be able to make sure the space-time box in each step of our iteration is deterministic and can be explicitly calculated.

Our construction starts with introducing the following families of independent Poisson processes: for all $x=\left(x_{1}, x_{2}\right)$ and $y=\left(y_{1}, y_{2}\right)$ that are nearest neighbors in $\mathcal{H}$ and $e_{x \rightarrow y}$ which is the oriented edge from $x$ to $y$, let

$$
\left\{N_{t}^{x \rightarrow y}, x, y \in \mathcal{H},\|x-y\|=1\right\}
$$

be a family of independent Poisson processes, where $N_{t}^{x \rightarrow y}$ has intensity $\sqrt{x_{2}} \vee 1$. Then let

$$
\left\{\tilde{N}_{t}^{x \rightarrow y}, x, y \in \mathcal{H},\|x-y\|=1\right\}
$$

be a family of independent Poisson process independent to $N_{t}$ with the same intensities. Now consider the space-time combination, $\mathcal{H} \times(-\infty, \infty)$. From each $x \in \mathcal{H}$, we draw a vertical infinite line to represent the double infinite time line at this site. Then for each $e_{x \rightarrow y}$, at any time $t$ such that $N_{t}^{x \rightarrow y}=N_{t-}^{x \rightarrow y}+1$, we draw an oriented arrow from $(x, t)$ to $(y, t)$. And at $t$ such that $\tilde{N}_{t}^{x \rightarrow y}=\tilde{N}_{t-}^{x \rightarrow y}+1$, we draw an oriented arrow from $(x,-t)$ to $(y,-t)$.

Remark 7. Although the construction of our particle system actually only depend on the transitions on the positive time line, by defining the transition for negative t's we are able to have better symmetry on the time reversal and thus formally simplify the proof.

We have an oriented random graph in the space-time combination. Then for any two points $\left(x, t_{1}\right)$ and $\left(x^{\prime}, t_{2}\right)$ with $t_{1}<t_{2}$, we define that $\left(x, t_{1}\right)$ and $\left(x^{\prime}, t_{2}\right)$ are connected or $\left(x, t_{1}\right) \rightarrow\left(x^{\prime}, t_{2}\right)$, if there is a (finite) path in the oriented random graph starting from $\left(x, t_{1}\right)$, that goes up vertically and follows the oriented edges ending at $\left(x^{\prime}, t_{2}\right)$. Then

Definition 1. For any $\bar{\xi}_{0} \in\{0,1\}^{\mathcal{H}}$, we define $\bar{\xi}_{t}$ such that for each $t \geq 0$ and $x \in \mathcal{H}, \bar{\xi}_{t}(x)=1$ if and only if there is a $x^{\prime}$ such that $\bar{\xi}_{0}\left(x^{\prime}\right)=1$ and $\left(x^{\prime}, 0\right) \rightarrow(x, t)$.

Once we prove that $\bar{\xi}_{t}$ is well defined, one can check that the conditions (i), (ii) are statisfied. And to show that $\bar{\xi}_{t}$ is well defined, it suffices to prove that in our oriented random graph, with probability one $(x, t)$ can be connected to only finitely many points $\left(x^{\prime}, 0\right)$ so one can determine explicitly whether any of them is occupied in the initial configuration. To be precise, for any $x \in \mathcal{H}$ and any $t, T \geq 0$, define subset

$$
R_{t, T}(x)=\{y \in \mathcal{H}, \text { s.t. }(y, T-t) \rightarrow(x, T)\}
$$

be the set of all possible ancestors of $\bar{\xi}_{T}(x)$ at time $T-t$, and we will write $R_{T}(x)$ in short of $R_{T, T}(x)$. According to the definition, it is easy to see that

$$
R_{t_{1}, T}(x) \underset{32}{\subset} R_{t_{2}, T}(x)
$$


for all $0 \leq t_{1} \leq t_{2}$ and $T \geq 0$, and that

$$
R_{T_{1}}(x) \subset R_{T_{2}}(x)
$$

for any $0 \leq T_{1} \leq T_{2}$. Thus, to show that Definition 1 is self-consistent, we only need to prove that

Lemma 6.1. With probability one we have $R_{T}(x)$ is finite for any $T>0$ and $x \in \mathcal{H}$.

Proof. Let

$$
\operatorname{Rad}_{t, T}(x)=\sup _{y \in R_{t, T}(x)}|x-y|
$$

be the radius of $R_{t, T}(x)$ and $\operatorname{Rad}_{T}(x)=\operatorname{Rad}_{T, T}(x)$. By (100), it is sufficient to prove that for each given $T>0$ and $x \in \mathcal{H}$ we have

$$
\operatorname{Rad}_{T}(x)<\infty
$$

almost surely. Then, we can take all rational numbers of $T$ 's and all $x \in \mathcal{H}$ which are both countable to get the lemma. Moreover, note that to show $P\left(\operatorname{Rad}_{T}(x)<\right.$ $\infty)=1$, it suffices to prove that for any $\epsilon>0$,

$$
P\left(\operatorname{Rad}_{T}(x)<\infty\right)>1-\epsilon
$$

For any given $T$ and $t \geq 0$ and $x=\left(x^{(1)}, x^{(2)}\right) \in \mathcal{H}$, note that $R_{t, T}(x)$ is the collection of all $x^{\prime}$ such that $\left(x^{\prime}, T-t\right)$ is connected to $(x, T)$. And for $\left(x^{\prime}, T-t\right)$ and $(x, T)$ to be connected, there must be a path between them, i.e., there must be a sequence of times $T-t \leq t_{1}<t_{2}<\cdots<t_{n} \leq T$ and $x^{\prime}=x_{0}, x_{1}, x_{2}, \cdots, x_{n}=x$ which is a nearest neighbor sequence in $\mathcal{H}$ such that

$$
N_{t_{i}}^{x_{i-1} \rightarrow x_{i}}=N_{t_{i}-}^{x_{i-1} \rightarrow x_{i}}+1
$$

if $t_{i} \geq 0$, or

$$
\tilde{N}_{-t_{i}}^{x_{i-1} \rightarrow x_{i}}=\tilde{N}_{-t_{i}-}^{x_{i-1} \rightarrow x_{i}}+1
$$

if $t_{i}<0$, for all $i=1,2, \cdots, n$. Thus it is easy to see that for any nearest neighbor path $x_{0}, x_{1}, x_{2}, \cdots, x_{n}=x$ in $\mathcal{H}$, it is open between $T-t$ and $T$ in our oriented random graph only if there is at least one transition at each edge along the path 
during this time interval. Thus we have

$$
\begin{aligned}
& P\left(\operatorname{Rad}_{t, T}(x) \geq n\right) \\
& \quad \leq P(\exists \text { an open path in }[T-t, T] \text { ending at } x \text { with length } n) \\
& \quad \leq \sum_{x_{0}, x_{1}, x_{2}, \cdots, x_{n} \in \mathcal{P}_{x, n}} \prod_{i=1}^{n} P\left(N_{T}^{x_{i-1} \rightarrow x_{i}}-N_{T-t}^{x_{i-1} \rightarrow x_{i}} \geq 1\right) \\
& \quad=\sum_{x_{0}, x_{1}, x_{2}, \cdots, x_{n} \in \mathcal{P}_{x, n}} \prod_{i=1}^{n}\left[1-e^{-t \sqrt{x_{i-1}^{(2)}}}\right] \\
& \leq t^{n} \sum_{x_{0}, x_{1}, x_{2}, \cdots, x_{n} \in \mathcal{P}_{x, n}} \sqrt{\prod_{i=1}^{n} x_{i-1}^{(2)}}
\end{aligned}
$$

where $\mathcal{P}_{x, n}$ is the collection of all nearest neighbor paths in $\mathcal{H}$ of length $n$ ending at $x$, and $x_{i-1}^{(2)}$ stands for the $y$-coordinate of $x_{i-1}$.

Remark 8. Without loss of generality, the inequalities above is written for $0 \leq$ $t \leq T$. By symmetry the same hold for $t>0$ and $T<0$. Note that even when $T>0$ and $T-t<0$, the total number of transitions of $N_{s}^{x_{i-1} \rightarrow x_{i}}$ in $s \in[0, T]$ plus the total number of transitions of $\tilde{N}_{s}^{x_{i-1} \rightarrow x_{i}}$ in $s \in[0, t-T]$ is again a Poisson random variable with intensity $t \sqrt{x_{i-1}^{(2)}}$. So (103) still holds.

Then note that $\left|\mathcal{P}_{x, n}\right| \leq 4^{n}$ and that for each $x_{0}, x_{1}, x_{2}, \cdots, x_{n}=x \in \mathcal{P}_{x, n}$, we have

Thus, we have

$$
x_{n-i}^{(2)} \leq x^{(2)}+i, i=0,1,2, \cdots, n .
$$

which implies that

$$
\sqrt{\prod_{i=1}^{n} x_{i-1}^{(2)}} \leq \sqrt{\prod_{i=1}^{n}\left(x^{(2)}+i\right)}
$$

$$
P\left(\operatorname{Rad}_{t, T}(x) \geq n\right) \leq(4 t)^{n} \sqrt{\prod_{i=1}^{n}\left(x^{(2)}+i\right)} .
$$

Now for each $\gamma \in(0,1 / 2)$, define

$$
M_{\gamma}=\sum_{k=0}^{\infty} k^{2 /(1-\gamma)} 2^{-k^{\gamma /(1-\gamma)}}<\infty .
$$

Now for any $\epsilon>0$ let

$$
N_{1}=\left\lfloor\frac{4\left(x^{(2)}\right)^{\gamma}}{1-\gamma}\right\rfloor
$$


and

$$
\delta_{1}=t_{1}=\frac{\epsilon}{64 M_{\gamma} \sqrt{x^{(2)}+N_{1}}}
$$

By (104), we have

$$
P\left(\operatorname{Rad}_{t_{1}, T}(x) \geq N_{1}\right) \leq \frac{\epsilon}{M_{\gamma}} 2^{-N_{1}} \leq \frac{\epsilon}{M_{\gamma}} k_{1}^{2 /(1-\gamma)} 2^{-k_{1}^{\gamma /(1-\gamma)}}
$$

where

$$
k_{1}=\left\lfloor\left(x^{(2)}\right)^{1-\gamma}\right\rfloor .
$$

The last inequality in (105) is a result that

$$
N_{1}=\left\lfloor\frac{4\left(x^{(2)}\right)^{\gamma}}{1-\gamma}\right\rfloor \geq\left(x^{(2)}\right)^{\gamma} \geq k_{1}^{\gamma /(1-\gamma)} .
$$

Then under event

$$
E_{1}=A_{1}=\left\{\operatorname{Rad}_{t_{1}, T}(x)<N_{1}\right\}
$$

we define

$$
\begin{gathered}
x^{(2), 2}=x^{(2)}+N_{1}, \\
N_{2}=\left\lfloor\frac{4\left(x^{(2), 2}\right)^{\gamma}}{1-\gamma}\right\rfloor, \\
\delta_{2}=\frac{\epsilon}{64 M_{\gamma} \sqrt{x^{(2), 2}+N_{2}}},
\end{gathered}
$$

and

$$
t_{2}=t_{1}+\delta_{2}
$$

Then define event

$$
A_{2}=\bigcap_{y \in B\left(x, x^{(2), 2}-x^{(2)}-1\right)}\left\{\operatorname{Rad}_{\delta_{2}, T-t_{1}}(y)<N_{2}\right\} .
$$

One can first see that by the same calculation as in (105)

$$
\begin{aligned}
P\left(A_{2}\right) & \geq 1-\sum_{y \in B\left(x, x^{(2), 2}-x^{(2)}-1\right)} P\left(\operatorname{Rad}_{\delta_{2}, T-t_{1}}(y) \geq N_{2}\right) \\
& \geq 1-\left(x^{(2), 2}\right)^{2} \frac{\epsilon}{16 M_{\gamma}} 2^{-k_{2}^{\gamma /(1-\gamma)}}
\end{aligned}
$$

where

Moreover, we have

$$
k_{2}=\left\lfloor\left(x^{(2), 2}\right)^{1-\gamma}\right\rfloor .
$$

while

$$
\left(x^{(2), 2}\right)^{2}=\left[\left(x^{(2), 2}\right)^{1-\gamma}\right]^{2 /(1-\gamma)}
$$

$$
\left(x^{(2), 2}\right)_{35}^{1-\gamma} \leq 2 k_{2} .
$$


Thus

$$
\begin{aligned}
P\left(A_{2}\right) & \geq 1-\left(2 k_{2}\right)^{2 /(1-\gamma)} \frac{\epsilon}{16 M_{\gamma}} 2^{-k_{2}^{\gamma /(1-\gamma)}} \\
& \geq 1-\frac{\epsilon}{M_{\gamma}} k_{2}^{2 /(1-\gamma)} 2^{-k_{2}^{\gamma /(1-\gamma)}} .
\end{aligned}
$$

Then note that for any $x \geq 1$, we have by calculus

while

$$
\left(x^{1-\gamma}+4\right)^{1 /(1-\gamma)}>x+\frac{4}{1-\gamma} x^{\gamma}
$$

We have that

$$
\begin{aligned}
\left(x^{1-\gamma}+1\right)^{1 /(1-\gamma)} & \leq x+\frac{1}{1-\gamma}\left(x^{1-\gamma}+1\right)^{\gamma /(1-\gamma)} \\
& \leq x+\frac{1}{1-\gamma}\left(2 x^{1-\gamma}\right)^{\gamma /(1-\gamma)} \\
& <x+\frac{2}{1-\gamma} x^{\gamma} \\
& <x+\left\lfloor\frac{4 x^{\gamma}}{1-\gamma}\right\rfloor .
\end{aligned}
$$

$$
\left(x^{(2), 2}\right)^{1-\gamma}=\left(x^{(2)}+N_{1}\right)^{1-\gamma} \in\left(\left(x^{(2)}\right)^{1-\gamma}+1,\left(x^{(2)}\right)^{1-\gamma}+4\right)
$$

and that

$$
k_{2}=\left\lfloor\left(x^{(2), 2}\right)^{1-\gamma}\right\rfloor \in\left[k_{1}+1, k_{1}+4\right) .
$$

Using exactly the same argument on

$$
x^{(2), 3}=x^{(2), 2}+N_{2},
$$

and

we have

$$
k_{3}=\left\lfloor\left(x^{(2), 3}\right)^{1-\gamma}\right\rfloor
$$

$$
k_{3} \in\left[k_{2}+1, k_{2}+4\right) .
$$

Then we note that event $A_{1}$ depends only on the transitions within $B\left(x, N_{1}\right) \times$ $\left[T-t_{1}, T\right]$, while event $A_{2}$ depends only on the transitions within $B\left(x, N_{1}+N_{2}\right) \times$ $\left[T-t_{2}, T-t_{1}\right]$. By the independence of increment in a Poisson processes, we have that $A_{1}$ independent to $A_{2}$, and thus for $E_{2}=A_{1} \cap A_{2}$,

$P\left(E_{2}\right)=P\left(A_{1}\right) P\left(A_{2}\right) \geq\left(1-\frac{\epsilon}{M_{\gamma}} k_{1}^{2 /(1-\gamma)} 2^{-k_{1}^{\gamma /(1-\gamma)}}\right) \cdot\left(1-\frac{\epsilon}{M_{\gamma}} k_{2}^{2 /(1-\gamma)} 2^{-k_{2}^{\gamma /(1-\gamma)}}\right)$.

Finally, recalling the definition of $\operatorname{Rad}_{t_{1}, T}$, one can immediately have under $E_{2}$

$$
\operatorname{Rad}_{t_{2}, T}(x)<x^{(2), 3}-x^{(2)}<\infty .
$$

Repeat such iteration, i.e., for all $n \geq 2$ let

$$
x^{(2), n}=x^{(2), n-1}+N_{n-1} \text {, }
$$




$$
\begin{gathered}
N_{n}=\left\lfloor\frac{4\left(x^{(2), n}\right)^{\gamma}}{1-\gamma}\right\rfloor, \\
\delta_{n}=\frac{\epsilon}{64 M_{\gamma} \sqrt{x^{(2), n}+N_{n}}}, \\
A_{n}=\bigcap_{y \in B\left(x, x^{(2), n}-x^{(2)}-1\right)}\left\{\operatorname{Rad}_{\delta_{n}, T-t_{n-1}}(y)<N_{n}\right\},
\end{gathered}
$$

and

Consider

$$
E_{n}=E_{n-1} \cap A_{n}
$$

Under $E_{\infty}$ we have for any $n \geq 1$,

$$
\operatorname{Rad}_{t_{n}, T}(x)<x^{(2), n+1}-x^{(2)}<\infty .
$$

At the same time

$$
t_{n}=\sum_{i=1}^{n} \delta_{i}=\sum_{i=1}^{n} \frac{\epsilon}{64 M_{\gamma} \sqrt{x^{(2), i}+N_{i}}}=\frac{\epsilon}{64 M_{\gamma}} \sum_{i=1}^{n} \frac{1}{\sqrt{x^{(2), i+1}}} .
$$

Moreover, by (109) we have for each $i$

$$
\left(x^{(2), i}\right)^{1-\gamma}=\left(x^{(2), i-1}+N_{i-1}\right)^{1-\gamma} \in\left(\left(x^{(2), i-1}\right)^{1-\gamma}+1,\left(x^{(2), i-1}\right)^{1-\gamma}+4\right)
$$

which together implies that

$$
\left(x^{(2), i}\right)^{1-\gamma} \leq\left(x^{(2)}\right)^{1-\gamma}+4 i .
$$

Combining (111)-(113) we have

$$
t_{n} \geq \frac{\epsilon}{64 M_{\gamma}} \sum_{i=1}^{n}\left[\left(x^{(2)}\right)^{1-\gamma}+4 i\right]^{-1 /(2-2 \gamma)} .
$$

Recalling that $\gamma \in(0,1 / 2), 1 /(2-2 \gamma)<1$, which implies that the series in (114) is divergent. So for any $T \geq 0$ there is a $n(T, \gamma, \epsilon)<\infty$ such that for all $n \geq n(T, \gamma, \epsilon), t_{n} \geq T$,

$$
\operatorname{Rad}_{T}(x) \subset \operatorname{Rad}_{t_{n}, T}(x) .
$$

Thus we have under event $E_{\infty}, \operatorname{Rad}_{T}(x)<\infty$. On the other hand, Noting that by the independence increment of Poisson processes, we have $A_{1}, A_{2}, \cdots$ gives a sequence of independent events, and that according to our iteration for each $i$

$$
P\left(A_{i}\right) \geq 1-\frac{\epsilon}{M_{\gamma}} k_{i}^{2 /(1-\gamma)} 2^{-k_{i}^{\gamma /(1-\gamma)}}>1-\epsilon
$$

with

$$
k_{i} \in\left[k_{i-1}+1, k_{i-1}+4\right) \text {. }
$$


Thus for sufficiently small $\epsilon$ such that for all $x \in(0, \epsilon), \log (1-x) \geq-2 x$ and any $n \geq 1$, we have

$$
P\left(E_{n}\right)-1 \geq \log \left(P\left(E_{n}\right)\right)=\log \left(\prod_{i=1}^{n} P\left(A_{i}\right)\right) \geq 2 \sum_{i=1}^{n}\left[P\left(A_{i}\right)-1\right] .
$$

By (115),

$$
P\left(E_{n}\right)-1 \geq-\frac{2 \epsilon}{M_{\gamma}} \sum_{i=1}^{n} k_{i}^{2 /(1-\gamma)} 2^{-k_{i}^{\gamma /(1-\gamma)}} .
$$

Then noting that by (116) $k_{i} \geq k_{i-1}+1$ and the fact that all $k_{i}$ 's are integers by definition, we have

$$
\sum_{i=1}^{n} k_{i}^{2 /(1-\gamma)} 2^{-k_{i}^{\gamma /(1-\gamma)}} \leq M_{\gamma}
$$

and thus

$$
P\left(E_{n}\right) \geq 1-2 \epsilon .
$$

Note that the right hand side of (117) is independent of $n$. We have $P\left(E_{\infty}\right) \geq$ $1-2 \epsilon$. And since $\epsilon$ is arbitrarily chosen, $P\left(\operatorname{Rad}_{T}(x)<\infty\right)=1$ which completes the proof of Lemma 6.1.

Thus the proof of Proposition 3 is complete.

With the proof of Proposition 3, one can easily apply the technique of Poisson thinning to define the following particle system where time is slowed down inhomogeneously and define a dominating process for the future stationary DLA model. I.e., we can consider the slower interacting particle system $\tilde{\xi}_{t}$ defined on $\{0,1\}^{\mathcal{H}}$ with transition rates as follows:

(i)' For each occupied site $x=\left(x_{1}, x_{2}\right) \in \mathcal{H}$ at time $t \geq 0$, it will try to give birth to each of its nearest neighbors at a Poisson rate of $\frac{\sqrt{x_{2}}}{\sqrt{t+1}}$.

(ii)' When $x$ attempts to give birth to its nearest neighbors $y$ already occupied, the birth is suppressed.

For $\tilde{\xi}_{t}$ we have

Corollary 2. The interacting particle system $\tilde{\xi}_{t} \in\{0,1\}^{\mathcal{H}}$ satisfying (i)' and (ii)' is well defined.

Proof. We construct $\tilde{\xi}_{t}$ with the same families of Poisson processes. Recall that in the proof of Proposition 3, for all $x=\left(x_{1}, x_{2}\right)$ and $y=\left(y_{1}, y_{2}\right)$ in $\mathcal{H}$ with $|x-y|=1$ and $e_{x \rightarrow y}$ which is the the oriented edge from $x$ to $y$, we have

$$
\left\{N_{t}^{x \rightarrow y}, x, y \in \underset{38}{\mathcal{H}},|x-y|=1\right\}
$$


be a family of independent Poisson process with intensity of $N_{t}^{x \rightarrow y}$ equals to $\sqrt{x_{2}}$. Moreover, for each $e_{x \rightarrow y}$, we define $\left\{U_{n}^{x \rightarrow y}\right\}_{n=1}^{\infty}$ be a i.i.d. sequence of random variables uniform on $[0,1]$. And we let the sequences for different edges independent to each other and also independent to the Poisson processes previously defined.

Now consider the space-time combination, $\mathcal{H} \times[0, \infty)$. From each $x \in \mathcal{H}$, we draw a vertical infinite half line to represent the time line at this site. Then for each $e_{x \rightarrow y}$, at any time $t$ such that $N_{t}^{x \rightarrow y}=n=N_{t-}^{x \rightarrow y}+1$, we draw an oriented arrow from $(x, t)$ to $(y, t)$ if $U_{n}^{x \rightarrow y}<1 / \sqrt{t+1}$. Thus we have another oriented random graph in the space-time combination which is a subset of the one we have for $\bar{\xi}_{t}$. By Proposition 3 we can see the following particle system is well defined.

Definition 2. For any $\tilde{\xi}_{0} \in\{0,1\}^{\mathcal{H}}$, we define $\tilde{\xi}_{t}$ such that for each $t \geq 0$ and $x \in \mathcal{H}, \tilde{\xi}_{t}(x)=1$ if and only if there is a $x^{\prime}$ such that $\bar{\xi}_{0}\left(x^{\prime}\right)=1$ and $\left(x^{\prime}, 0\right)$ is connected to $(x, t)$ in the new smaller oriented random graph.

6.2. Proof of Theorem 5. By Theorem 1 we have seen that for any $B, x \in B \backslash L_{0}$ and any $\vec{e}=x \rightarrow y$ with $\|x-y\|=1$,

$$
H_{B}(\vec{e}) \leq H_{B}(x) \leq C \sqrt{x_{2}}
$$

for some $C>1$. Moreover, by (18), if $x_{2}=0$,

$$
H_{B}(\vec{e}) \leq H_{B}(x) \leq 1 .
$$

We construct our DLA model on $\mathcal{H}$ as follows: First, recall that

$$
\left\{N_{t}^{x \rightarrow y}, x, y \in \mathcal{H},\|x-y\|=1\right\}
$$

is a family of independent Poisson processes, such that the intensity of $N_{t}^{x \rightarrow y}$ equals to $\sqrt{x_{2}}$ and that $\left\{U_{n}^{x \rightarrow y}\right\}_{n=1}^{\infty}$ is an i.i.d. sequence of random variables uniform on $[0,1]$ independent to the Poisson processes. Let $\bar{A}_{0}=\{(0,0)\}$, and for any $t>0$,

- If there is an $\vec{e}=x \rightarrow y$ such that $x \in \bar{A}_{t-}$ and $y \notin \bar{A}_{t-}$, where $N_{t}^{x \rightarrow y}=n$ and $N_{t-}^{x \rightarrow y}=n-1$, we let $\bar{A}_{t}=\bar{A}_{t-} \cup\{y\}$ if

$$
U_{n}^{x \rightarrow y} \leq \frac{H_{\bar{A}_{t-}}(\vec{e})}{C \sqrt{x_{2}}} .
$$

- Otherwise, $\bar{A}_{t}=\bar{A}_{t-}$.

To prove Theorem 5 , we first need to show

Lemma 6.2. For each time $t, \bar{A}_{t}$ above is with probability 1 well defined and finite.

Proof. To prove Lemma 6.2, we construct an event with probability one such that $\bar{A}_{t}$ is well defined and finite under this event. For any $x \in \mathcal{H}$ and any $0 \leq t<T$, define subset

$$
I_{t, T}(x)=\{y \in \mathcal{H}, \text { s.t. }(x, t) \rightarrow(y, T)\}
$$


and let

$$
\mathcal{I}_{t, T}(x)=\sup _{y \in I_{t, T}(x)}\|x-y\| .
$$

Following exactly the same argument as in Lemma 6.1, we have with probability one

$$
\mathcal{I}_{0, C t}(0)<\infty
$$

Under $\left\{\mathcal{I}_{0, C t}(0)<\infty\right\}$ one can easily put all of the finite Poisson transitions within the space time box $\mathcal{I}_{0, t}(0) \times[0, t]$ in order and construct $\bar{A}_{t}$ explicitly over finite steps. Moreover, by definition we can always have $\bar{A}_{t} \subset I_{0, t}(0)$ thus $\bar{A}_{t}$ is finite.

Let $A_{t}=\bar{A}_{C t}$, then it is easy to check $A_{t}$ has the same dynamic as in Theorem 5 while being almost surely well defined and finite at the same time. Now, to finish the proof of Theorem 5, we again follow the argument as in Lemma 6.1.

Remark 9. The proof of Theorem 6 actually also contains all that is needed here (and more). Thus we will not present the details of basically the same thing for a third time.

By (113), (114) and (117) we have for any $\gamma \in(0,1 / 2)$ and $\epsilon>0$, there are constants $0<c_{\gamma}, C<\infty$, and deterministic sequences

$$
t_{n}^{\epsilon, \gamma} \geq c_{\gamma} \epsilon n^{\frac{1-2 \gamma}{2-2 \gamma}}, n=1,2, \cdots
$$

and

$$
R_{n}^{\epsilon, \gamma} \leq C n^{\frac{1}{1-\gamma}}, n=1,2, \cdots
$$

and event $E_{\infty}^{\epsilon, \gamma}$ such that $P\left(E_{\infty}^{\epsilon, \gamma}\right) \geq 1-2 \epsilon$ and that under $E_{\infty}^{\epsilon, \gamma}$

$$
\mathcal{I}_{0, t_{n}^{\epsilon, \gamma}}(0) \leq R_{n}^{\epsilon, \gamma}, \forall n=1,2, \cdots
$$

With (119) we have for any $t \geq t_{1}$, let

$$
n_{t}=\sup \left\{k: t_{k} \leq t\right\} \text {. }
$$

Then under event $E_{\infty}^{\epsilon, \gamma}$

$$
\mathcal{I}_{0, t}(0) \leq R_{n_{t}+1}^{\epsilon, \gamma} \leq C\left(n_{t}+1\right)^{\frac{1}{1-\gamma}}
$$

which implies that

$$
\mathcal{I}_{0, t}(0) t^{\frac{-2}{1-2 \gamma}} \leq 4 C\left(c_{\gamma} \epsilon\right)^{\frac{-2}{1-2 \gamma}}<\infty
$$

Note that the right hand side of (120) is independent to the choice of $t$, and that $P\left(E_{\infty}^{\epsilon, \gamma}\right) \geq 1-2 \epsilon$ for all $\epsilon$. we have for any given $\gamma \in(0,1 / 2)$, with probability one

$$
\limsup _{t \rightarrow \infty}\left\|A_{t}\right\| t^{\frac{-2}{1-2 \gamma}} \leq \limsup _{t \rightarrow \infty} \mathcal{I}_{0, C t}(0)(C t)^{\frac{-2}{1-2 \gamma}}<\infty .
$$

Finally note that the choice of $\gamma$ is arbitrary and that $2 /(1-\gamma) \rightarrow 2$ as $\gamma \rightarrow 0$. The proof of Theorem 5 is complete. 
6.3. Proof of Theorem 6. To prove (14), since we have $A_{t}=\bar{A}_{C t} \subset I_{0, C t}(0)$, it is sufficient to show for any $t \geq 0$ and integer $m \geq 1$

$$
E\left[\mathcal{I}_{0, t}(0)^{m}\right]<\infty .
$$

The proof here is similar to the one for Lemma 6.1. However, since some more delicate estimates on the upper bounds of probabilities are needed, we still provide a detailed proof for the completeness of this paper.

Recall (103), we have for any $t$ and $n$,

$$
\begin{aligned}
& P\left(\mathcal{I}_{0, t}(0) \geq n\right) \\
& \leq P(\exists \text { an open path in }[0, t] \text { starting at } 0 \text { with length } n) \\
& \leq \sum_{x_{0}, x_{1}, x_{2}, \cdots, x_{n} \in \mathcal{P}_{n, 0}} \prod_{i=1}^{n} P\left(N_{T}^{x_{i-1} \rightarrow x_{i}}-N_{T-t}^{x_{i-1} \rightarrow x_{i}} \geq 1\right) \\
& =\left(1-e^{-t}\right) \sum_{x_{0}, x_{1}, x_{2}, \cdots, x_{n} \in \mathcal{P}_{n, 0}} \prod_{i=1}^{n-1}\left[1-e^{-t \sqrt{x_{i}^{(2)}}}\right] \\
& \quad \leq t^{n} \sum_{x_{0}, x_{1}, x_{2}, \cdots, x_{n} \in \mathcal{P}_{n, 0}} \sqrt{\prod_{i=1}^{n} x_{i}^{(2)}} .
\end{aligned}
$$

Here we use $\mathcal{P}_{n, 0}$ to denote the collection of all nearest neighbor paths starting at 0 with length $n$. Then note that $\left|\mathcal{P}_{n, 0}\right| \leq 4^{n}$ and that for each $0=x_{0}, x_{1}, x_{2}, \cdots, x_{n} \in$ $\mathcal{P}_{n, 0}$, we have

$$
x_{i}^{(2)} \leq i, i=0,1,2, \cdots, n
$$

Thus, we have

which implies that

$$
\sqrt{\prod_{i=1}^{n} x_{i}^{(2)}} \leq \sqrt{n !}
$$

$$
P\left(\mathcal{I}_{0, t}(0) \geq n\right) \leq(4 t)^{n} \sqrt{n !}
$$

Now for each $\gamma \in(0,1 / 2)$, define

$$
M_{\gamma}=\sum_{k=0}^{\infty} k^{2 /(1-\gamma)} 2^{-k^{\gamma /(1-\gamma)}}<\infty .
$$

Now for any $\epsilon>0$ let

and

$$
N_{1}=\left\lfloor\frac{4 m}{1-\gamma}\right\rfloor \geq 4 m
$$

$$
\delta_{1}=t_{1}=\frac{\epsilon}{64 M_{\gamma} \sqrt{N_{1}}} .
$$


By (124), we have

$$
\begin{aligned}
P\left(\mathcal{I}_{0, t_{1}}(0) \geq N_{1}\right) & \leq\left(\frac{\epsilon}{16 M_{\gamma} \sqrt{N_{1}}}\right)^{N_{1}} \sqrt{N_{1} !} \\
& \leq \frac{\epsilon^{4 m}}{M_{\gamma}} 16^{-N_{1}} \leq \frac{\epsilon^{4 m}}{M_{\gamma}} k_{1}^{2 /(1-\gamma)} 2^{-k_{1}^{\gamma /(1-\gamma)}}
\end{aligned}
$$

where $k_{1}=1$. Then under event

$$
E_{1}=A_{1}=\left\{\mathcal{I}_{0, t_{1}}(0)<N_{1}\right\}
$$

we define

$$
\begin{gathered}
x^{(2), 2}=1+N_{1}, \\
N_{2}=\left\lfloor\frac{4 m\left(x^{(2), 2}\right)^{\gamma}}{1-\gamma}\right\rfloor \geq 4 m \\
\delta_{2}=\frac{\epsilon}{64 M_{\gamma} \sqrt{x^{(2), 2}+N_{2}}},
\end{gathered}
$$

and

Then define event

$$
t_{2}=t_{1}+\delta_{2}
$$

$$
A_{2}=\bigcap_{y \in B\left(0, x^{(2), 2}-1\right)}\left\{\mathcal{I}_{t_{1}, t_{2}}(y)<N_{2}\right\}
$$

One can first see that by the same calculation as in (105)

$$
\begin{aligned}
P\left(A_{2}\right) & \geq 1-\sum_{y \in B\left(0, x^{(2), 2}-1\right)} P\left(\mathcal{I}_{t_{1}, t_{2}}(y) \geq N_{2}\right) \\
& \geq 1-4\left(x^{(2), 2}\right)^{2}\left(\frac{\epsilon}{16 M_{\gamma} \sqrt{x^{(2), 2}+N_{2}}}\right)^{N_{2}} \sqrt{\prod_{j=x^{(2), 2}-1}^{x^{(2), 2}+N_{2}-2} j} \\
& \geq 1-\left(x^{(2), 2}\right)^{2} \frac{\epsilon^{4 m}}{16 M_{\gamma}} 2^{-k_{2}^{\gamma /(1-\gamma)}}
\end{aligned}
$$

where

$$
k_{2}=\left\lfloor\left(x^{(2), 2}\right)^{1-\gamma}\right\rfloor .
$$

The last inequality in (126) results from

$$
N_{2}=\left\lfloor\frac{4 m\left(x^{(2), 2}\right)^{\gamma}}{1-\gamma}\right\rfloor \geq\left(x^{(2), 2}\right)^{\gamma} \geq k_{1}^{\gamma /(1-\gamma)} .
$$

Moreover, we have

$$
\left.\left(x^{(2), 2}\right)^{2}=\underset{42}{\left(x^{(2), 2}\right)^{1-\gamma}}\right]^{2 /(1-\gamma)}
$$


while

$$
\left(x^{(2), 2}\right)^{1-\gamma} \leq 2 k_{2}
$$

Thus

$$
\begin{aligned}
P\left(A_{2}\right) & \geq 1-\left(2 k_{2}\right)^{2 /(1-\gamma)} \frac{\epsilon^{4 m}}{16 M_{\gamma}} 2^{-k_{2}^{\gamma /(1-\gamma)}} \\
& \geq 1-\frac{\epsilon^{4 m}}{M_{\gamma}} k_{2}^{2 /(1-\gamma)} 2^{-k_{2}^{\gamma /(1-\gamma)}} .
\end{aligned}
$$

Then note that for any $x \geq 1$, we have by calculus

$$
\left(x^{1-\gamma}+4 m\right)^{1 /(1-\gamma)}>x+\frac{4 m}{1-\gamma} x^{\gamma}
$$

while

We have that

$$
\begin{aligned}
\left(x^{1-\gamma}+1\right)^{1 /(1-\gamma)} & \leq x+\frac{1}{1-\gamma}\left(x^{1-\gamma}+1\right)^{\gamma /(1-\gamma)} \\
& \leq x+\frac{1}{1-\gamma}\left(2 x^{1-\gamma}\right)^{\gamma /(1-\gamma)} \\
& <x+\frac{2}{1-\gamma} x^{\gamma} \\
& <x+\left\lfloor\frac{4 m x^{\gamma}}{1-\gamma}\right\rfloor .
\end{aligned}
$$

$$
\left(x^{(2), 2}\right)^{1-\gamma}=\left(1+N_{1}\right)^{1-\gamma}=\left(1+\left\lfloor\frac{4 m \cdot 1^{\gamma}}{1-\gamma}\right\rfloor\right)^{1-\gamma} \in(2,1+4 m)
$$

and that

$$
k_{2}=\left\lfloor\left(x^{(2), 2}\right)^{1-\gamma}\right\rfloor \in\left(k_{1}+1, k_{1}+4 m\right)
$$

since $k_{1}=1$. Then using exactly the same argument on

$$
x^{(2), 3}=x^{(2), 2}+N_{2},
$$

and

we have

$$
k_{3}=\left\lfloor\left(x^{(2), 3}\right)^{1-\gamma}\right\rfloor,
$$

$$
\begin{aligned}
\left(x^{(2), 3}\right)^{1-\gamma}=\left(x^{(2), 2}+N_{2}\right)^{1-\gamma} & =\left(x^{(2), 2}+\left\lfloor\frac{4 m\left(x^{(2), 2}\right)^{\gamma}}{1-\gamma}\right\rfloor\right)^{1-\gamma} \\
& \in\left(\left(x^{(2), 2}\right)^{1-\gamma}+1,\left(x^{(2), 2}\right)^{1-\gamma}+4 m\right)
\end{aligned}
$$

and thus

$$
k_{3} \in\left(k_{2}+1, k_{2}+4 m\right) .
$$

Then we note that the event $A_{1}$ depends only on the transitions within $B\left(0, N_{1}\right) \times$ $\left[0, t_{1}\right]$, while event $A_{2}$ depends only on the transitions within $B\left(0, N_{1}+N_{2}\right) \times\left[t_{1}, t_{2}\right]$. 
By the independence of Poisson process increments, we have that $A_{1}$ is independent to $A_{2}$, and thus for $E_{2}=A_{1} \cap A_{2}$,

$P\left(E_{2}\right)=P\left(A_{1}\right) P\left(A_{2}\right) \geq\left(1-\frac{\epsilon^{4 m}}{M_{\gamma}} k_{1}^{2 /(1-\gamma)} 2^{-k_{1}^{\gamma /(1-\gamma)}}\right) \cdot\left(1-\frac{\epsilon^{4 m}}{M_{\gamma}} k_{2}^{2 /(1-\gamma)} 2^{-k_{2}^{\gamma /(1-\gamma)}}\right)$.

Finally, recalling the definition of $\mathcal{I}_{0, t}$, one can immediately have under $E_{2}$

$$
\mathcal{I}_{0, t_{2}}(0)<x^{(2), 3}<\infty .
$$

Repeat the iteration above, i.e., for all $n \geq 3$ let

$$
\begin{gathered}
x^{(2), n}=x^{(2), n-1}+N_{n-1}, \\
N_{n}=\left\lfloor\frac{4 m\left(x^{(2), n}\right)^{\gamma}}{1-\gamma}\right\rfloor, \\
\delta_{n}=\frac{\epsilon}{64 M_{\gamma} \sqrt{x^{(2), n}+N_{n}}}, \\
A_{n}=t_{n-1}+\delta_{n}, \\
\bigcap_{y \in B\left(0, x^{(2), n}-1\right)}\left\{\mathcal{I}_{t_{n-1}, t_{n}}(y)<N_{n}\right\},
\end{gathered}
$$

and

$$
E_{n}=E_{n-1} \cap A_{n}
$$

Consider

$$
E_{\infty}^{\epsilon}=\bigcap_{n=1}^{\infty} A_{n}
$$

Under $E_{\infty}^{\epsilon}$ we have for any $n \geq 1$,

$$
\mathcal{I}_{0, t_{n}}(x)<x^{(2), n+1}<\infty .
$$

At the same time

$$
t_{n}=\sum_{i=1}^{n} \delta_{i}=\sum_{i=1}^{n} \frac{\epsilon}{64 M_{\gamma} \sqrt{x^{(2), i}+N_{i}}}=\frac{\epsilon}{64 M_{\gamma}} \sum_{i=1}^{n} \frac{1}{\sqrt{x^{(2), i+1}}} .
$$

Moreover, by (129) we have for each $i$

$$
\left(x^{(2), i}\right)^{1-\gamma}=\left(x^{(2), i-1}+N_{i-1}\right)^{1-\gamma} \in\left(\left(x^{(2), i-1}\right)^{1-\gamma}+1,\left(x^{(2), i-1}\right)^{1-\gamma}+4 m\right)
$$

which together implies that

$$
\left(x^{(2), i}\right)^{1-\gamma} \leq 4 i m .
$$

Combining (131)-(133) we have

$$
t_{n} \geq \frac{\epsilon}{64 M_{\gamma}} \sum_{i=1}^{n}(4 i m)^{-1 /(2-2 \gamma)} .
$$


Recalling that $\gamma \in(0,1 / 2), 1 /(2-2 \gamma)<1$, which implies that the series in (134) is divergent. So for any $t \geq 0$ there is an $n_{0}<\infty$ such that for all $n \geq n_{0}$, $t_{n} \geq t$, and that $t_{n_{0}-1}<t$.

$$
\mathcal{I}_{0, t}(0) \leq \mathcal{I}_{0, t_{n_{0}}}(0) .
$$

And by (130) and (133), under $E_{\infty}^{\epsilon}$,

$$
\mathcal{I}_{0, t_{n}}(0)<x^{(2), n+1} \leq\left[4 m\left(n_{0}+1\right)\right]^{1 /(1-\gamma)} .
$$

Thus we have under event $E_{\infty}^{\epsilon}$,

$$
\mathcal{I}_{0, t}(0) \leq\left[4 m\left(n_{0}+1\right)\right]^{1 /(1-\gamma)} \leq(8 m)^{1 /(1-\gamma)} \cdot n_{0}^{1 /(1-\gamma)}
$$

On the other hand,

$$
t \geq t_{n_{0}-1} \geq \frac{\epsilon}{64 M_{\gamma}} \sum_{i=1}^{n_{0}-1}(4 m \cdot i)^{-1 /(2-2 \gamma)} \geq \frac{c \epsilon}{64 M_{\gamma}(4 m)^{1 /(2-2 \gamma)}} n_{0}^{(1-2 \gamma) /(2-2 \gamma)}
$$

Combining (135) and (136), we have under event $E_{\infty}^{\epsilon}$ there is a constant $C_{m, \gamma}$ depending on $m$ and $\gamma$ but independent to $\epsilon$ such that

$$
\mathcal{I}_{0, t}(0) \leq C_{m, \gamma} \epsilon^{-2 /(1-\gamma)} t^{2 /(1-2 \gamma)} .
$$

Note that by the independence of Poisson processes increments, we have that $A_{1}, A_{2}, \cdots$ gives a sequence of independent events. And according to (126) and the construction in our iteration, we have for each $i$

$$
P\left(A_{i}\right) \geq 1-\frac{\epsilon^{4 m}}{M_{\gamma}} k_{i}^{2 /(1-\gamma)} 2^{-k_{i}^{\gamma /(1-\gamma)}}>1-\epsilon
$$

with

$$
k_{i}=\left\lfloor\left(x^{(2), i}\right)^{1-\gamma}\right\rfloor \in\left[k_{i-1}+1, k_{i-1}+4 m\right) .
$$

Thus for sufficiently small $\epsilon$ such that for all $x \in(0, \epsilon), \log (1-x) \geq-2 x$ and any $n \geq 1$, we have

$$
P\left(E_{n}\right)-1 \geq \log \left(P\left(E_{n}\right)\right)=\log \left(\prod_{i=1}^{n} P\left(A_{i}\right)\right) \geq 2 \sum_{i=1}^{n}\left[P\left(A_{i}\right)-1\right] .
$$

By (138),

$$
P\left(E_{n}\right)-1 \geq-\frac{2 \epsilon^{4 m}}{M_{\gamma}} \sum_{i=1}^{n} k_{i}^{2 /(1-\gamma)} 2^{-k_{i}^{\gamma /(1-\gamma)}} .
$$

Then noting that by (139) $k_{i} \geq k_{i-1}+1$ and the fact that all $k_{i}$ 's are integers by definition, we have

and thus

$$
\sum_{i=1}^{n} k_{i}^{2 /(1-\gamma)} 2^{-k_{i}^{\gamma /(1-\gamma)}} \leq M_{\gamma}
$$

$$
P\left(E_{n}\right) \geq \underset{45}{1-2 \epsilon^{4 m}}
$$


Note that the right hand side of $(140)$ is independent of $n$. We have $P\left(E_{\infty}^{\epsilon}\right) \geq$ $1-2 \epsilon^{4 m}$. Now let

$$
\epsilon_{j}=\left(\frac{1}{j}\right)^{\frac{1-r}{2 m}} .
$$

Then we have for each $j$ sufficiently large,

$$
\begin{aligned}
P\left(\frac{\mathcal{I}_{0, t}(0)^{m}}{\left(C_{m, \gamma}\right)^{m} t^{2 m /(1-2 \gamma)}}>\epsilon_{j}^{-2 m /(1-\gamma)}\right) & =P\left(\mathcal{I}_{0, t}(0)>C_{m, \gamma} \epsilon_{j}^{-2 /(1-\gamma)} t^{2 /(1-2 \gamma)}\right) \\
& \leq 1-P\left(E_{\infty}^{\epsilon_{j}}\right) \leq 2 \epsilon_{j}^{4 m}
\end{aligned}
$$

and thus

$$
P\left(\frac{\mathcal{I}_{0, t}(0)^{m}}{\left(C_{m, \gamma}\right)^{m} t^{2 m /(1-2 \gamma)}}>j\right) \leq 2\left(\frac{1}{j}\right)^{2(1-\gamma)} .
$$

Noting that $\gamma<1 / 2$ and thus $2(1-\gamma)>1$,

$$
\sum_{n=1}^{\infty} P\left(\frac{\mathcal{I}_{0, t}(0)^{m}}{\left(C_{m, \gamma}\right)^{m} t^{2 m /(1-2 \gamma)}}>j\right)<\infty
$$

which implies that $E\left[\mathcal{I}_{0, t}(0)^{m}\right]<\infty$.

\section{ACKNOWLEDGMENTS}

We would like to thank Itai Benjamini, Noam Berger, Marek Biskup, Rick Durrett and Gady Kozma for fruitful discussions in the initiation of this project.

\section{REFERENCES}

[1] T. Antunović and E. B. Procaccia. Stationary eden model on cayley graphs. The Annals of Applied Probability, 27(1):517-549, 2017.

[2] I. Benjamini and A. Yadin. Upper bounds on the growth rate of diffusion limited aggregation. arXiv preprint arXiv:1705.06095, 2017.

[3] N. Berger, J. J. Kagan, and E. B. Procaccia. Stretched IDLA. ALEA Lat. Am. J. Probab. Math. Stat., 11(1):471-481, 2014.

[4] R. Durrett. Ten lectures on particle systems. In Lectures on probability theory (Saint-Flour, 1993), volume 1608 of Lecture Notes in Math., pages 97-201. Springer, Berlin, 1995.

[5] A. Dvoretzky and P. Erdös. Some problems on random walk in space. In Proceedings of the Second Berkeley Symposium on Mathematical Statistics and Probability, 1950, pages 353-367. University of California Press, Berkeley and Los Angeles, 1951.

[6] P. Erdös and S. J. Taylor. Some problems concerning the structure of random walk paths. Acta Math. Acad. Sci. Hungar., 11:137-162. (unbound insert), 1960.

[7] H. Kesten. Hitting probabilities of random walks on $\mathbf{Z}^{d}$. Stochastic Process. Appl., 25(2):165$184,1987$.

[8] H. Kesten. How long are the arms in DLA? J. Phys. A, 20(1):L29-L33, 1987.

[9] H. Kesten. Upper bounds for the growth rate of DLA. Phys. A, 168(1):529-535, 1990.

[10] E. B. Procaccia and Y. Zhang. On sets of zero stationary harmonic measure. arXiv preprint arXiv:1711.01013, 2017. 
[11] E. B. Procaccia and Y. Zhang. Two dimensional stationary dla. in preparation, 2018.

[12] T. A. Witten and L. M. Sander. Diffusion-limited aggregation. Phys. Rev. B (3), 27(9):56865697, 1983.

(Eviatar B. Procaccia ${ }^{1}$ ) Texas A\&M University

$U R L$ : www.math.tamu.edu/ procaccia

E-mail address: eviatarp@gmail.com

(Yuan Zhang) TeXAs A\&M University

URL: http://www . math.tamu .edu/ yzhang1988/

E-mail address: yzhang1988@math.tamu.edu

${ }^{1}$ Research supported by NSF grant DMS-1407558 